\title{
Heterotic effective action and duality symmetries revisited
}

\author{
Olaf Hohm, ${ }^{a}$ Ashoke Sen ${ }^{b}$ and Barton Zwiebach ${ }^{a}$ \\ ${ }^{a}$ Center for Theoretical Physics, Massachusetts Institute of Technology, \\ Cambridge, MA 02139, U.S.A. \\ ${ }^{b}$ Harishchandra Research Institute, \\ Chhatnag Road, Jhusi, Allahabad 211019, India \\ E-mail: ohohm@mit.edu, sen@hri.res.in, zwiebach@mit.edu
}

ABSTRACT: The dimensional reduction of heterotic supergravity with gauge fields truncated to the Cartan subalgebra exhibits a continuous $O(d, d+16 ; \mathbb{R})$ global symmetry, related to the $O(d, d+16 ; \mathbb{Z})$ T-duality of heterotic strings on a $d$-torus. The $O(d, d+16 ; \mathbb{R})$ symmetry is not present, however, if the supergravity reduction is done including the full set of $E_{8} \times E_{8}$ or $\mathrm{SO}(32)$ gauge fields. We analyze which duality symmetries are realized to all orders in $\alpha^{\prime}$ in the proper effective field theories for the massless string states. We find a universal $O(d, d ; \mathbb{R})$ symmetry, also predicted by double field theory. We confirm this by giving a novel formulation of the dimensionally reduced supergravity in terms of $O(d, d)$ multiplets, and we discuss cases of symmetry enhancement.

KEYwords: Superstrings and Heterotic Strings, Supergravity Models, Global Symmetries, String Duality

ARXIV EPRINT: 1411.5696 


\section{Contents}

1 Introduction 1

2 String theoretic argument $\quad 4$

3 Torus compactifcation with non-abelian gauge groups $\quad 8$

3.1 Remarks on flux quantization 8

$\begin{array}{lll}3.2 & \text { Torus compactification of heterotic supergravity } & 9\end{array}$

4 Compactified theory in terms of $O(d, d+K)$ multiplets $\quad 14$

5 Compactified theory in terms of $O(d, d)$ multiplets $\quad 18$

$\begin{array}{ll}5.1 \text { Introducing } O(d, d) \text { field multiplets } & 19\end{array}$

$\begin{array}{ll}5.2 \text { Covariant action } & 22\end{array}$

6 Conclusions $\quad 26$

\section{Introduction}

The T-duality properties of heterotic string theory were studied in the seminal works of Narain [1] and Narain, Sarmadi, and Witten [2]. The T-duality group $O(d, d+16 ; \mathbb{Z})$ arises from a compactification on a $d$-dimensional torus that includes Wilson lines in the Cartan subgroup of the gauge group. Duality symmetries have a counterpart in continuous global symmetries of the low-energy action for the massless fields [3-6]. A particularly clear discussion of this relationship was given by Maharana and Schwarz in [7]. (For earlier results see $[8,9]$.) In order to explain the global $O(d, d+16 ; \mathbb{R})$ of the low-energy limit of compactified heterotic strings they considered heterotic supergravity with the gauge group truncated to the maximal Cartan subgroup. They performed dimensional reduction and displayed the expected global symmetry of the reduced theory. With the development of double field theory formulations [10-14] of the low-energy limits of string theories, the manifest display of global duality symmetries and the effect of $\alpha^{\prime}$ corrections is now the subject of renewed interest [15-27]. Motivated by this, we revisit here some aspects of the continuous T-duality symmetry of the heterotic string effective action.

Maharana and Schwarz (MS) truncate the higher dimensional heterotic supergravity theory to the Cartan subgroup before performing the reduction. In fact, the $O(d, d+16 ; \mathbb{R})$ symmetry is not present upon reduction if one includes any non-abelian gauge group. This is puzzling because, after all, the gauge group in heterotic string theory is $E_{8} \times E_{8}$ or $\mathrm{SO}(32)$. The main goals of this paper are to clarify, on general string-theoretic grounds, which duality symmetry we should expect for the effective spacetime theory of the massless fields to any order in $\alpha^{\prime}$, and to exhibit this symmetry in a manifest form. 
We use the symmetries of S-matrix elements of massless states to explain that, to all orders in $\alpha^{\prime}$, the effective action for the massless fields has a universal global $O(d, d ; \mathbb{R})$ symmetry. ${ }^{1}$ The arguments, which are an elaboration of those in $[5,6]$, do not preclude possible enhancements at points of the moduli space with reduced sets of massless fields. We also perform the direct dimensional reduction of the non-abelian action. Since dimensional reduction can be viewed physically as compactification on tori without any field dependence on the toroidal coordinates, we verify that the non-abelian gauge field reduction is not constrained by flux quantization. ${ }^{2}$ The resulting action (3.32) is similar to that of MS, with new non-abelian gauge-covariant couplings and potential terms. The $O(d, d ; \mathbb{R})$ symmetry of this action is not manifest (although it is certainly present). Introducing Wilson lines for the Cartan gauge fields corresponds to giving expectation values to the scalars that arise from the internal components of the Cartan gauge fields. ${ }^{3}$ In doing so, all scalars and gauge fields arising from the non-Cartan gauge fields acquire masses via a Higgs mechanism. They must be dropped to describe the proper effective action, given that we do not include massive Kaluza-Klein modes nor massive string modes. Restricted to the massless fields, the action now becomes the MS action with an enhanced $O(d, d+16 ; \mathbb{R})$ symmetry.

The general presence of a global $O(d, d ; \mathbb{R})$ symmetry to lowest order in $\alpha^{\prime}$ is also implied by the double field theory formulation of heterotic supergravity $[10,11,15,16]$. The doubled formulation uses $O(d, d+K ; \mathbb{R})$ multiplets, with $K$ the dimension of the non-abelian gauge group. This $O(d, d+K ; \mathbb{R})$ group, however, is not a symmetry. As discussed in [15], the actual global symmetry depends on the gauge group but contains at least $O(d, d)$. Guided by these results we cast the dimensionally reduced supergravity, including all non-abelian gauge fields, into an $O(d, d+K ; \mathbb{R})$ 'covariant' form (see (4.1)).

Since only $O(d, d)$ is always an actual symmetry, it is desirable to formulate the theory in terms of $O(d, d)$ multiplets. Let us stress that this is a non-trivial problem because the symmetries are non-linearly realized. As the main technical result of this paper we present such a formulation. We write the action in terms of an $O(d, d)$ valued 'generalized metric' $\mathcal{H}$ and a Lie algebra valued $O(d, d)$ vector $\mathcal{C}$. Specifically, the scalars arising out of the internal components of the metric, 2-form-field, and non-abelian gauge vectors, denoted by $G, B$, and $a$, respectively, are encoded in the following fields:

$$
O(d, d) \text { field content: } \quad \mathcal{H}_{M N}, \quad \mathcal{C}_{M}{ }^{\alpha},
$$

where $M, N, \ldots$ are fundamental $O(d, d)$ indices and $\alpha, \beta$ denote the adjoint gauge group

\footnotetext{
${ }^{1}$ We exclude the massless non-abelian fields which could arise e.g. when some of the radii take self-dual values. If we keep these non-abelian fields then the continuous duality symmetry of the tree effective action of massless fields could be further reduced.

${ }^{2}$ Due to the commutator terms in non-abelian field strengths, even constant gauge field configurations give rise to fluxes.

${ }^{3}$ Given a field with a space-time index, we will call the internal components those where the index takes value on the compact coordinates. We will call the external components those where the index takes value on the non-compact coordinates. For a gauge field, for example, the internal components represent scalars of the lower-dimensional theory and the external components comprise a gauge field of the lowerdimensional theory.
} 
indices. These fields satisfy the following constraints, written in matrix notation:

$$
\mathcal{H} \eta \mathcal{H}=\eta, \quad(1+\mathcal{H} \eta) \mathcal{C}=0,
$$

where $\eta$ is the $O(d, d)$ invariant metric. The first constraint simply states the familiar property of the generalized metric, the second is an $O(d, d)$ covariant constraint on $\mathcal{C}$. Given the first constraint, the second one is in fact a projector condition that cuts half of the degrees of freedom in $\mathcal{C}$. The fields can be parametrized in terms of $G, B$ and $a$. For $\mathcal{H}$ we find

$$
\mathcal{H}=\left(\begin{array}{cc}
\bar{G}^{-1} & -\bar{G}^{-1} B \\
B \bar{G}^{-1} & \bar{G}-B \bar{G}^{-1} B
\end{array}\right),
$$

while $\mathcal{C}$ can be written as

$$
\mathcal{C}=\frac{1}{2}\left(\begin{array}{c}
-\bar{G}^{-1} a^{T} \kappa \\
-B \bar{G}^{-1} a^{T} \kappa+a^{T} \kappa
\end{array}\right),
$$

where $\kappa$ denotes the Cartan-Killing metric of the gauge group. For $\mathcal{H}$ this is the familiar form, except that the internal metric $G$ is redefined with a contribution from the internal $E_{8} \times E_{8}$ or $\mathrm{SO}(32)$ gauge field components:

$$
\bar{G} \equiv G+\frac{1}{2} a^{T} \kappa a .
$$

The action for dimensionally reduced heterotic supergravity in these new variables is given in (5.43). We note that the redefinition (1.5) is compatible with the findings of refs. [32] and [33], which determined the Buscher rules for the heterotic theory with a single circle direction and found that such a redefinition naturally occurs. Interestingly, a redefinition of the type (1.5) also featured in [34], for reasons seemingly unrelated to T-duality.

So far we have discussed two cases. One is heterotic string compactifications with non-zero Wilson lines for the 16 gauge fields in the Cartan subalgebra of the gauge algebra $G$, resulting in a moduli space $O(d, d+16) / O(d) \times O(d+16)$ and $O(d, d+16 ; \mathbb{Z})$ dualities. The other one represents the case where there is not a single Wilson line. Here the moduli space is $O(d, d) / O(d) \times O(d)$ and we have $O(d, d ; \mathbb{Z})$ dualities. The intermediate situation, however, is also of interest. Letting superscripts denote rank, consider a subgroup $G^{(r)} \times$ $G^{(16-r)}$ of the rank 16 gauge group $G$. We can then imagine a compactification with Wilson lines for the Cartan gauge fields $\mathrm{U}(1)^{(16-r)}$ of the second factor. The moduli space of such compactification is $O(d, d+16-r) / O(d) \times O(d+16-r)$ and the full string duality group is $O(d, d+16-r ; \mathbb{Z})$. In this case the non-Cartan gauge fields of the second factor, as well as the gauge fields outside the $G^{(r)} \times G^{(16-r)}$ subgroup, acquire masses. The massless fields, apart from those from the gravitational multiplet, are the internal and external components of the full $G^{(r)}$ gauge fields and the internal and external components of the $U(1)^{(16-r)}$ gauge fields. According to the argument given in section 2, the effective field theory of such fields will have a global $O(d, d+16-r ; \mathbb{R})$ duality symmetry to all orders in $\alpha^{\prime}$. The two-derivative version of this action is given by the same expression (5.43), with $\mathcal{H}$ now interpreted as a symmetric $O(d, d+16-r)$ matrix and $\mathcal{C}$ interpreted as a $(2 d+16-r) \times \operatorname{dim} G^{(r)}$ matrix, transforming as a vector of $O(d, d+16-r)$ and as an adjoint of $G^{(r)}$. 
This paper is organized as follows. In section 2 we give the string theoretic arguments for the global duality symmetries of the effective field theories of heterotic massless fields. The power of this argument is that it works to all orders in $\alpha^{\prime}$. We turn in section 3 to the torus compactification of the heterotic supergravity action, including the effect of the non-abelian gauge fields. In section 4 we recast this action in terms of a formal $O(d, d+K)$ symmetry, with $K$ the dimension of the gauge group. Section 5 gives a rewriting of this theory in terms of $O(d, d)$ multiplets, making this symmetry manifest. In section 6 we discuss the possible relevance of our analysis for double field theory formulations of heterotic strings that include $\alpha^{\prime}$ corrections.

\section{String theoretic argument}

We shall review the string theoretic argument for the existence of $O(d, d)$ symmetry in the presence of non-abelian gauge fields [6]. This argument is valid in classical string theory to all orders in $\alpha^{\prime}$. The main idea is to determine the symmetries of the action in a consistently truncated sector by studying the symmetries of the S-matrix in the same sector. We shall then combine this with the obvious symmetries of the effective action the $G L(d)$ symmetry associated with the linear transformation of the compact coordinates and the shift symmetry of the 2-form field - to determine the full symmetry group of the truncated effective action. The latter symmetries are not visible as symmetries of the S-matrix since they are typically spontaneously broken in a given background.

The theory under consideration is heterotic string theory and the truncation we are interested in requires all fields to be independent of $d$ of the spatial coordinates. The corresponding S-matrix will involve external states which carry zero momentum along the $d$ directions but has no further restrictions. Also since we shall be interested in the classical effective action where we have integrated out all the massive string fields, it is sufficient to examine the S-matrix with massless external states only.

While we shall consider a general set of external states subject to the condition of independence of the $d$ spatial coordinates, we shall work in a special background left invariant by a large subset of the duality symmetries: the two-form field and all gauge fields are zero and the metric is the diagonal unit metric. Working with such special background may seem a strong assumption but it is not so. Once we have determined the symmetries of the S-matrix and translated them into a symmetry statement for the effective action around the special background, the symmetry must also hold for the effective action in the more general backgrounds that can be obtained by switching on fields within the truncated class. This is true even if the symmetry is spontaneously broken in the new background and is therefore not a symmetry of the S-matrix. In our case, since the massless set of states include those for the internal components of the metric, two-form, and non abelian gauge fields, the general backgrounds for which these have expectation values are covered in the argument.

Let us denote by $X^{\mu}$ the space-time coordinates on which the fields are allowed to depend and by $Y^{m}$ the $d$ coordinates on which the fields do not depend. We also denote by $\psi^{\mu}$ and $\chi^{m}$ their fermionic partners. The vertex operators of the massless bosonic states 
in the minus-one picture are then given by

$$
\begin{array}{ll}
\psi^{\mu} \bar{\partial} X^{\nu} e^{i k \cdot X} e^{-\phi}, & \\
\psi^{\mu} \bar{\partial} Y^{n} e^{i k \cdot X} e^{-\phi}, & \chi^{m} \bar{\partial} X^{\mu} e^{i k \cdot X} e^{-\phi}, \\
\chi^{m} \bar{\partial} Y^{n} e^{i k \cdot X} e^{-\phi}, & \\
\psi^{\mu} \bar{J}^{\alpha} e^{i k \cdot X} e^{-\phi}, & \chi^{m} \bar{J}^{\alpha} e^{i k \cdot X} e^{-\phi},
\end{array}
$$

where $\phi$ is the scalar arising from bosonization of the superconformal ghost system [35] and the $\bar{J}^{\alpha}$ are dimension $(1,0)$ vertex operators in the anti-holomorphic sector describing the $E_{8} \times E_{8}$ or $\mathrm{SO}(32)$ currents. The vertex operators on the first line include those for the lower-dimensional metric, two-form, and dilaton. On the second line we have those for the lower-dimensional abelian gauge fields that arise from the metric and the two-form. On the third line we have the vertex operators for the scalar fields that arise from the internal components of the metric and two form. On the last line we have the vertex operators for the lower-dimensional non-abelian gauge fields (first term) and for the scalars arising from the internal components of the non-abelian gauge fields. None of the vertex operators in the above list carry momentum or winding along the $d$ compact coordinates.

The S-matrix will be computed from the correlation function of these vertex operators together with suitable insertions of picture changing operators. The (holomorphic) picture changing operator with picture number plus one has the form

$$
-e^{\phi}\left(\psi_{\mu} \partial X^{\mu}+\chi_{m} \partial Y^{m}\right)+\cdots
$$

where $\cdots$ denotes terms involving only ghost sector fields. In the following we shall focus specifically on the tree level S-matrix which requires correlation functions of the conformal field theory on the sphere. Now the key observations are the following:

1. In computing sphere correlation functions of operators in (2.1) and picture changing operators, we can treat the $d$ internal coordinates associated with the fields $Y^{m}$ as if they were non-compact. The compactness of these coordinates will affect the correlation functions of vertex operators carrying non-zero momentum or winding number along these directions as well as higher-genus correlation functions of the vertex operators given in (2.1), but not the correlation functions of the vertex operators in (2.1) on the sphere.

2. The correlation functions of the vertex operators (2.1), picture changing operators, and the additional ghost insertions needed to provide the correct integration measure over the moduli space of the punctured sphere can be expressed as a sum of correlators each of which factorizes into three factors: a correlator involving $\left(Y^{m}, \chi^{m}\right)$ 's, a correlator involving the $\bar{J}^{\alpha}$ s, and a correlator involving the other conformal fields.

3. On the sphere the correlation functions of the $Y^{n}$ 's satisfy holomorphic factorization. As a result a correlation function involving the $\left(\partial Y^{m}, \bar{\partial} Y^{m}\right)$ 's further factorizes into a correlation function involving $\partial Y^{m}$ and one involving $\bar{\partial} Y^{n}$. This allows us to express the correlation functions of vertex operators in (2.1), picture changing operators and 
other ghost insertions as sum of terms each of which has four parts: a correlator involving $\left(\partial Y^{m}, \chi^{m}\right)$ 's, a correlator involving $\bar{\partial} Y^{m}$ 's, a correlator involving the $\bar{J}^{\alpha}$ 's, and a correlator involving the other conformal fields.

4. Given point 1, we can compute the required correlators in a theory where $Y^{m}$ 's are non-compact. In this case both the world-sheet theory for the $\left(Y^{m}, \chi^{m}\right)$ fields and the picture changing operator are $O(d)$ invariant, where $O(d)$ acts as simultaneous rotation of the $Y^{m}$ 's and $\chi^{m}$ 's. Thus the factor in the correlator involving the $\left(\partial Y^{m}, \chi^{m}\right)$ 's is $O(d)$ invariant. Furthermore due to holomorphic factorization the factor involving the $\bar{\partial} Y^{n}$ 's must also have an independent $O(d)$ symmetry. As needed, this symmetry is also a (trivial) symmetry of the picture changing operator, which does not involve the operator $\bar{\partial} Y^{n}$. In summary, we have an $O(d) \times O(d)$ symmetry.

5. The vertex operators given in (2.1) provide a representation of this $O(d) \times O(d)$ symmetry, 1.e. the action of $O(d) \times O(d)$ does not take us outside this list. Thus the correlation functions and hence the S-matrix elements must have $O(d) \times O(d)$ symmetry.

6. If we expand the tree level S-matrix elements of massless states in string theory in powers of $\alpha^{\prime}$, then to any given order in $\alpha^{\prime}$ we can find a general coordinate invariant effective action whose tree level S-matrix elements coincide with those computed from string theory. The $O(d) \times O(d)$ symmetry of the S-matrix elements then implies that the effective action that reproduces this S-matrix must also have $O(d) \times O(d)$ symmetry. Furthermore at the linearized level the action of this symmetry on the massless fields can be read out from their action on the vertex operators.

7. The effective action that reproduces the tree level S-matrix elements of massless fields in toroidally compactified string theory can be regarded as the restriction of a general covariant action in $9+1$ dimensions to field configurations independent of $d$ coordinates. Since a general linear transformation on the $d$ coordinates preserves the property that the field configuration is independent of these $d$ coordinates, it must be a symmetry of the resulting action. More precisely, in a generic theory of this kind, $G L(d)$ is only a symmetry of the equation of motion and its $\operatorname{SL}(d)$ subgroup is a symmetry of the action since the $\sqrt{\operatorname{det} G}$ factor in the Lagrangian density is not $G L(d)$ invariant. In tree level string theory, however, the change in $\sqrt{\operatorname{det} G}$ can be cancelled by a shift in the dilaton field making $G L(d)$ a symmetry of the effective action.

Note that not all of this $G L(d)$ symmetry preserves the background and thus not all of it is a symmetry of the S-matrix. The $O(d)$ subgroup of $G L(d)$ describing the rotation of the $y$-coordinates preserves the background and is a symmetry of the S-matrix. ${ }^{4}$ This can be identified as the diagonal subgroup of the $O(d) \times O(d)$ symmetry discussed above.

\footnotetext{
${ }^{4}$ Even this $O(d)$ symmetry is broken once we take into account the periodic identification of the internal coordinates, but this effect is not visible at tree-level string theory when we have an effective theory for states carrying zero momenta along the internal directions.
} 
8. The full symmetry of the effective action must include both $O(d) \times O(d)$ and $G L(d)$. The diagonal subgroup of $O(d) \times O(d)$ lies within $G L(d)$; so the number of independent generators we get this way is $d^{2}$ from $G L(d)$ and $d(d-1) / 2$ from one of the $O(d)$ 's. To this we must add the shift symmetry of the two-form fields; these are also manifest symmetries of the dimensionally reduced action since in all terms in the effective action the two-form appears with an exterior derivative acting on it. These are parametrized by $d \times d$ anti-symmetric matrices and give $d(d-1) / 2$ more independent generators. Together they account for the $d(2 d-1)$ independent generators of $O(d, d)$. Thus $O(d, d)$ must be a symmetry of the effective action to all orders in the $\alpha^{\prime}$ expansion.

We would like to remark that instead of working with the S-matrix elements we could also work with any string field theory of heterotic NS fields, such as [28]. In that case our arguments will directly imply the $O(d) \times O(d)$ symmetry of the string field theory action when we restrict the string fields to carry zero momentum along $d$ of the spatial directions. Since the effective action is obtained from this by integrating out the massive string fields followed by possible field redefinitions, it will inherit the $O(d) \times O(d)$ symmetry. Combining this with $G L(d)$ and shift symmetries we can prove the $O(d, d)$ symmetry of the effective action.

The S-matrix argument can be easily generalized to consider a truncation where we allow only gauge fields inside a subgroup $G \times \mathrm{U}(1)^{p}$ of $E_{8} \times E_{8}$ or $\mathrm{SO}(32)$ to be switched on. Let $\bar{J}^{\alpha^{\prime}}$ denote the currents for $G$ and we represent the $p$ abelian currents by $i \bar{\partial} U^{k}$, with $k=1, \ldots, p$, and $U^{k}$ new chiral world-sheet scalar fields. ${ }^{5}$ In this case the list of operators in (2.1) is modified:

$$
\begin{aligned}
& \psi^{\mu} \bar{\partial} X^{\nu} e^{i k \cdot X} e^{-\phi}, \\
& \psi^{\mu} \bar{\partial} \mathcal{Y}^{\bar{n}} e^{i k \cdot X} e^{-\phi}, \\
& \chi^{m} \bar{\partial} \mathcal{Y}^{\bar{n}} e^{i k \cdot X} e^{-\phi},
\end{aligned}
$$

with

$$
\bar{\partial} \mathcal{Y}^{\bar{n}}=\left\{\bar{\partial} Y^{1}, \ldots, \bar{\partial} Y^{d}, \bar{\partial} U^{1}, \ldots \bar{\partial} U^{p}\right\}, \quad \bar{n}=1, \ldots, d+p .
$$

The main effect has been to include the U(1) currents into an extended version $\bar{\partial} \mathcal{Y}$ of the $\bar{\partial} Y$ conformal fields. Having truncated the gauge group, we now have less massless states.

We can now repeat the above arguments. The correlation functions factorize into correlators involving the $\bar{J}^{\alpha^{\prime}}$ and the rest. As before, the correlators involving $\left(\partial Y^{m}, \psi^{m}\right)$ 's are $O(d)$ invariant. Furthermore the correlators involving $\bar{\partial} \mathcal{Y}^{\bar{n}}$ 's factor from the rest and have an $O(d+p)$ symmetry. Thus the S-matrix and the effective action has $O(d) \times O(d+p)$ symmetry to all orders in $\alpha^{\prime}$. We need to combine this with the manifest $G L(d)$ symmetry, the shift symmetry of the 2 -form fields parametrized by $d \times d$ anti-symmetric matrices and the shift symmetry of the internal components of the $p$ gauge fields parametrized by $d \times p$

\footnotetext{
${ }^{5}$ Strictly speaking the fields $U^{k}$ do not exist as conformal fields but the currents $\bar{\partial} U^{k}$ do, and all our manipulations will involve only the currents.
} 
matrices. ${ }^{6}$ Taking into account that the diagonal $O(d)$ of $O(d) \times O(d+p)$ is included in $G L(d)$, we get altogether

$$
d^{2}+\frac{1}{2}(d+p)(d+p-1)+\frac{1}{2} d(d-1)+d p=\frac{1}{2}(2 d+p)(2 d+p-1)
$$

generators, which is the right number of generators of $O(d, d+p)$.

\section{Torus compactifcation with non-abelian gauge groups}

We perform the torus compactification of the spacetime action of heterotic strings for the massless fields, to zeroth order in $\alpha^{\prime}$, but including all non-abelian gauge fields for a group $G$. Our goal is to investigate which global duality symmetry emerges once the massive Kaluza-Klein modes are truncated out. In the first subsection we make some general remarks on torus compactification or dimensional reduction and the nature (or rather absence) of flux quantization conditions. Then we present the technical details of the Kaluza-Klein reduction, which will be used in the following sections in order to write the action in terms of $O(d, d+\operatorname{dim} G)$ and $O(d, d)$ multiplets, respectively.

\subsection{Remarks on flux quantization}

Dimensional reduction, as distinguished from compactification, is generally understood as a procedure in which a theory formulated in a $D$-dimensional space-time is used to construct a $D-p$ dimensional field theory with $0<p<D$. This is done by assuming that all fields are independent of $p$ spatial dimensions and evaluating the original action with this assumption. The nature of the extra dimensions is left unspecified and any volume of the extra dimensions is taken to be a constant that can be absorbed in the normalization of the action, sometimes as a rescaling of a coupling constant.

In order for dimensional reduction to produce a theory that is physically related to the original higher-dimensional theory, one must specify the shape of the extra dimensions; one must do compactification. The simplest compact $p$-dimensional space in which fields can consistently be set to be constant is the $p$-dimensional torus $T^{p}$. Even this is not completely obvious for the cause of gauge fields, as we will discuss below. The dimensionally reduced theory is then obtained from the compactified theory by ignoring all Kaluza-Klein excitations that arise from field configurations in which fields depend on the compact space. Thus we view dimensional reduction as compactification on tori.

When an abelian gauge theory is defined on a torus there are configurations where the gauge fields are not constant over the torus and as a result there are non-vanishing field strengths. The total flux associated with an abelian field strength is quantized because only then space-dependent gauge fields on the torus are well-defined globally.

For compactification on a torus we will consider the ansatz in which all higherdimensional non-abelian gauge fields $\hat{A}_{\hat{\mu}}{ }^{\alpha}$ are independent of the toroidal directions $y^{m}$.

\footnotetext{
${ }^{6}$ Although the Chern-Simons terms are not invariant under the shift symmetry of the gauge fields, the three form field strength can be made invariant under this transformation by including a compensating transformation of the 2 -form fields.
} 
Letting $a_{m}{ }^{\alpha}$ denote the components of the non-abelian gauge fields along toroidal directions, the field strength $F_{m n}{ }^{\alpha}$ along toroidal directions is then given by

$$
F_{m n}{ }^{\alpha}=\partial_{m} a_{n}^{\alpha}-\partial_{n} a_{m}^{\alpha}+f^{\alpha}{ }_{\beta \gamma} a_{m}{ }^{\beta} a_{n}^{\gamma}=f_{\beta \gamma}^{\alpha} a_{m}{ }^{\beta} a_{n}^{\gamma} .
$$

It is now clear that the dimensional reduction hypothesis of coordinate independence can lead to non-vanishing non-abelian field strengths. This could not happen for abelian gauge fields, where only spatial dependence can lead to field strengths. Moreover, unless the fields $a_{m}{ }^{\alpha}$ satisfy unusual constraints, the associated fields strengths will actually take arbitrary continuous values. We claim that there is no condition on the constant non-abelian gauge fields on the torus, and no quantization of the resulting fluxes. This is simply because constant gauge fields on a torus are globally well-defined regardless of their value: they require no gauge transformation to patch up as we traverse any non contractible closed loop on the torus. This means that we can perform the dimensional reduction without topological complications.

It should be noted that in general spatially varying non-abelian gauge field configurations may require a quantization condition to be globally well defined, resulting in quantized fluxes. Here we see that non-abelian field strengths arising from spatial derivatives are not on the same footing as field strengths arising from the commutator term (for which there is no quantization, if the connections are spatially constant). Indeed, one can find a simple example of non-abelian $\mathrm{SU}(2)$ gauge fields where gauge fields with spatial dependence and gauge field without spatial dependence give rise to the same field strength. These configurations are not even locally gauge equivalent.

\subsection{Torus compactification of heterotic supergravity}

We now perform the explicit compactification starting from the heterotic spacetime action. Even though this theory is defined in 10 space-time dimensions we shall keep our analysis slightly more general by taking the initial space-time dimension to be $D$. Denoting the $D$-dimensional objects and indices by hats, the action is given by

$$
S=\int \mathrm{d}^{D} x \sqrt{-\hat{g}} e^{-2 \hat{\phi}}\left[\hat{R}+4(\partial \hat{\phi})^{2}-\frac{1}{12} \hat{H}^{\hat{\mu} \hat{\nu} \hat{\rho}} \hat{H}_{\hat{\mu} \hat{\nu} \hat{\rho}}-\frac{1}{4} \hat{F}^{\hat{\mu} \hat{\nu} \alpha} \hat{F}_{\hat{\mu} \hat{\nu} \alpha}\right]
$$

The Einstein-Hilbert and dilaton terms are unchanged compared to the abelian case, but the field strengths are now

$$
\begin{aligned}
& \hat{H}_{\hat{\mu} \hat{\nu} \hat{\rho}}=3\left(\partial_{[\hat{\mu}} \hat{b}_{\hat{\nu} \hat{\rho}]}-\hat{A}_{[\hat{\mu}}^{\alpha} \partial_{\hat{\nu}} \hat{A}_{\hat{\rho}] \alpha}-\frac{1}{3} f_{\alpha \beta \gamma} \hat{A}_{\hat{\mu}}^{\alpha} \hat{A}_{\hat{\nu}}^{\beta} \hat{A}_{\hat{\rho}}^{\gamma}\right), \\
& \hat{F}_{\hat{\mu} \hat{\nu}}{ }^{\alpha}=\partial_{\hat{\mu}} \hat{A}_{\hat{\nu}}^{\alpha}-\partial_{\hat{\nu}} \hat{A}_{\hat{\mu}}{ }^{\alpha}+f_{\beta \gamma}{ }^{\alpha} \hat{A}_{\hat{\mu}}^{\beta} \hat{A}_{\hat{\nu}}^{\gamma}
\end{aligned}
$$

where $\alpha, \beta$ are the adjoint indices of the Lie algebra associated with the gauge group. With Lie algebra generators $T_{\alpha}$ we have $\left[T_{\alpha}, T_{\beta}\right]=f_{\alpha \beta}{ }^{\gamma} T_{\gamma}$, where $f_{\alpha \beta}{ }^{\gamma}$ are the structure constants. For semisimple gauge algebras we use the Cartan-Killing metric

$$
\kappa_{\alpha \beta} \equiv-\operatorname{tr}\left(\operatorname{ad} T_{\alpha} \circ \operatorname{ad} T_{\beta}\right)=-f_{\alpha \gamma} \delta f_{\beta \delta}^{\gamma},
$$


to lower indices, leading to $f_{\alpha \beta \gamma} \equiv f_{\alpha \beta}{ }^{\rho} \kappa_{\rho \gamma}$ that is totally antisymmetric. The inverse of $\kappa_{\alpha \beta}$ exists and is written as $\kappa^{\alpha \beta}$. Note also that the overall normalization of $\kappa$ is not important as it can be absorbed into a rescaling of the metric, dilaton and two form fields. If the gauge group is of the form $G^{\prime} \times \mathrm{U}(1)^{p}$ with the Lie algebra of $G^{\prime}$ semisimple, then $\kappa$ is defined to be a block diagonal matrix containing the $p \times p$ identity matrix $I_{p}$ and the Cartan-Killing metric $\kappa^{\prime}$ for $G^{\prime}$ :

$$
\text { For } G^{\prime} \times \mathrm{U}(1)^{p}: \quad \kappa \equiv\left(\begin{array}{cc}
I_{p} & 0 \\
0 & \kappa^{\prime}
\end{array}\right)
$$

This $\kappa$ matrix is still invertible.

We perform the dimensional reduction by splitting the coordinates into non-compact and compact ones, corresponding to a toroidal background

$$
\mathbb{R}^{n-1,1} \times T^{d}, \quad D=n+d .
$$

Specifically, we write $x^{\hat{\mu}}=\left(x^{\mu}, y^{m}\right)$, corresponding to the index split

$$
\hat{\mu}=(\mu, m), \quad \hat{a}=(a, \underline{a}),
$$

where the second equation indicates the splitting of the flat (Lorentz) indices. The Lorentz metric is

$$
\hat{\eta}_{\hat{a} \hat{b}}=\left(\begin{array}{cc}
\eta_{a b} & 0 \\
0 & \delta_{\underline{a} \underline{b}}
\end{array}\right)
$$

with $\eta_{a b}$ for the noncompact directions and $\delta_{\underline{a}} \underline{b}$ for the compact ones. The Kaluza-Klein ansatz for the vielbein $\hat{e}_{\hat{\mu}}^{\hat{a}}$ (and its inverse $\hat{e}_{\hat{a}}{ }_{\hat{\mu}}$ ) is

$$
\hat{e}_{\hat{\mu}}^{\hat{a}}=\left(\begin{array}{cc}
e_{\mu}{ }^{a} & A_{\mu}^{(1) m} E_{m}{ }^{\underline{a}} \\
0 & E_{m^{\underline{a}}}
\end{array}\right), \quad \hat{e}_{\hat{a}}^{\hat{\mu}}=\left(\begin{array}{cc}
e_{a}^{\mu}-e_{a}^{\nu} A_{\nu}^{(1) m} \\
0 & E_{\underline{a}}{ }^{m}
\end{array}\right),
$$

where $e_{\mu}{ }^{a}$ and $e_{a}{ }^{\mu}$ are inverses of each other, $E_{m} \underline{\underline{a}}$ and $E_{\underline{a}}{ }^{m}$ are inverses of each other, and $A_{\mu}^{(1) m}$ denote a collection of Kaluza-Klein vectors labelled by $m$. We define

$$
g_{\mu \nu} \equiv e_{\mu}{ }^{a} e_{\nu} \eta_{a b}, \quad G_{m n} \equiv E_{m} \underline{a} E_{n} \underline{\underline{b}} \delta_{\underline{a}} \underline{\underline{b}} .
$$

In terms of these we have, with $\hat{\mu}=(\mu, m)$ and $\hat{\nu}=(\nu, n)$,

$$
\hat{g}_{\hat{\mu} \hat{\nu}} \equiv \hat{e}_{\hat{\mu}} \hat{a}_{\hat{\nu}} \hat{b}_{\hat{\nu}} \hat{\eta}_{\hat{a} \hat{b}}=\left(\begin{array}{cc}
g_{\mu \nu}+A_{\mu}^{(1) m} G_{m n} A_{\nu}^{(1) n} & A_{\mu}^{(1) k} G_{k n} \\
A_{\nu}^{(1) k} G_{k m} & G_{m n}
\end{array}\right) \text {. }
$$

In order to obtain canonically normalized and manifestly gauge invariant kinetic terms in the reduced theory we have to perform a number of field redefinitions for the vectors and two-forms. The general prescription, as also employed by Maharana-Schwarz, is to define components of the $D$-dimensional fields with flat indices and then to 'un-flatten' with the 
lower-dimensional vielbein. This is best explained using an object with a single index, as the generalization to multiple indices is trivial. Given an object $\hat{W}_{\hat{\mu}}$ with $\hat{\mu}=(\mu, m)$ we define

$$
\begin{aligned}
W_{m} & \equiv \hat{W}_{m} \\
W_{\mu} & \equiv e_{\mu}{ }^{a} \hat{e}_{a}^{\hat{\nu}} \hat{W}_{\hat{\nu}}=e_{\mu}{ }^{a} \hat{W}_{a} .
\end{aligned}
$$

Using the explicit form of the vielbein we get

$$
W_{\mu}=e_{\mu}^{a}\left(\hat{e}_{a}^{\nu} \hat{W}_{\nu}+\hat{e}_{a}{ }^{n} \hat{W}_{n}\right)=\hat{W}_{\mu}-e_{\mu}{ }^{a} e_{a}{ }^{\nu} A_{\nu}^{(1) n} \hat{W}_{n}
$$

and therefore

$$
W_{\mu}=\hat{W}_{\mu}-A_{\mu}^{(1) n} \hat{W}_{n} .
$$

When we deal with multiple indices we apply the rule in (3.12) to each of the indices. The logic behind the rule is that one can quickly verify that

$$
\begin{aligned}
W^{\mu} W_{\mu} & \equiv g^{\mu \nu} W_{\mu} W_{\nu}=\eta^{a b} \hat{W}_{a} \hat{W}_{b} \equiv \hat{W}^{a} \hat{W}_{a}, \\
W^{m} W_{m} & \equiv G^{m n} W_{m} W_{n}=\delta^{\underline{a}} \underline{W_{\underline{a}}} \hat{W}_{\underline{b}},
\end{aligned}
$$

and this leads to

$$
\hat{W}^{\hat{\mu}} \hat{W}_{\hat{\mu}} \equiv \hat{g}^{\hat{\mu} \hat{\nu}} \hat{W}_{\hat{\mu}} \hat{W}_{\hat{\nu}}=\hat{\eta}^{\hat{a} \hat{b}} \hat{W}_{\hat{a}} \hat{W}_{\hat{b}}=W^{\mu} W_{\mu}+W^{m} W_{m},
$$

giving a very simple way to expand contracted full-dimensional indices, without off-diagonal metric contributions involving bare Kaluza-Klein vectors.

We turn now to the decomposition of the gauge kinetic terms. For the field strength $\hat{H}_{\hat{\mu} \hat{\nu} \hat{\rho}}$ we have, for example,

$$
H_{\mu m n} \equiv e_{\mu}{ }^{a} \hat{e}_{a}^{\hat{\nu}} \hat{H}_{\hat{\nu} m n} \quad \rightarrow \quad H_{\mu m n} \equiv \hat{H}_{\mu m n}-A_{\mu}^{(1) k} \hat{H}_{k m n}
$$

For the full set of components we find

$$
\begin{aligned}
H_{m n k} & \equiv \hat{H}_{m n k}, \\
H_{\mu m n} & \equiv \hat{H}_{\mu m n}-A_{\mu}^{(1) k} \hat{H}_{k m n}, \\
H_{\mu \nu m} & \equiv \hat{H}_{\mu \nu m}-2 A_{[\mu}^{(1) n} \hat{H}_{\nu] m n}+A_{\mu}^{(1) n} A_{\nu}^{(1) k} \hat{H}_{m n k}, \\
H_{\mu \nu \rho} & \equiv \hat{H}_{\mu \nu \rho}-3 A_{[\mu}^{(1) m} \hat{H}_{\nu \rho] m}+3 A_{[\mu}^{(1) m} A_{\nu}^{(1) n} \hat{H}_{\rho] m n}-A_{\mu}^{(1) m} A_{\nu}^{(1) n} A_{\rho}^{(1) k} \hat{H}_{m n k} .
\end{aligned}
$$

Analogous redefinitions are needed for the Yang-Mills field strength:

$$
\begin{aligned}
F_{m n}{ }^{\alpha} & \equiv \hat{F}_{m n}{ }^{\alpha}, \\
F_{\mu m}{ }^{\alpha} & \equiv \hat{F}_{\mu m}{ }^{\alpha}+A_{\mu}^{(1) n} \hat{F}_{m n}{ }^{\alpha}, \\
F_{\mu \nu}{ }^{\alpha} & \equiv \hat{F}_{\mu \nu}{ }^{\alpha}+2 A_{[\mu}^{(1) m} \hat{F}_{\nu] m}{ }^{\alpha}+A_{\mu}^{(1) m} A_{\nu}^{(1) n} \hat{F}_{m n}{ }^{\alpha} .
\end{aligned}
$$

Our formula (3.16) makes the expansion of kinetic terms trivial. It follows that the Yang-Mills kinetic term decomposes as

$$
-\frac{1}{4} \hat{F}^{\hat{\mu} \hat{\nu} \alpha} \hat{F}_{\hat{\mu} \hat{\nu} \alpha}=-\frac{1}{4} F^{\mu \nu \alpha} F_{\mu \nu \alpha}-\frac{1}{2} F^{\mu m \alpha} F_{\mu m \alpha}-\frac{1}{4} F^{m n \alpha} F_{m n \alpha} .
$$


Similarly, for the two-form kinetic term:

$$
-\frac{1}{12} \hat{H}^{\hat{\mu} \hat{\nu} \hat{\rho}} \hat{H}_{\hat{\mu} \hat{\nu} \hat{\rho}}=-\frac{1}{12} H^{\mu \nu \rho} H_{\mu \nu \rho}-\frac{1}{4} H^{\mu \nu m} H_{\mu \nu m}-\frac{1}{4} H^{\mu m n} H_{\mu m n}-\frac{1}{12} H^{m n k} H_{m n k} .
$$

The index contractions above are done using the metrics $g_{\mu \nu}$ and $G_{m n}$. In the two equations above, the new terms compared to MS are those with purely internal coordinates: $F_{m n \alpha}^{2}$ and $H_{m n k}^{2}$. These vanish when fields are $y$ independent and the gauge group is abelian but are non-zero when the gauge group is non-abelian. These terms simply give the potential:

$$
-V=-\frac{1}{12} H^{m n k} H_{m n k}-\frac{1}{4} F^{m n \alpha} F_{m n \alpha} .
$$

The little less trivial part of the computation is to express the above field strengths in terms of the gauge potentials that are redefined as well in order to exhibit the non-abelian symmetry in conventional form. For the gauge potentials the original field $\hat{A}_{\hat{\mu}}{ }^{\alpha}$ yield fields $a_{m}{ }^{\alpha}$ and $A_{\mu}{ }^{\alpha}$ from the postulated rule:

$$
\begin{aligned}
a_{m}{ }^{\alpha} & \equiv \hat{A}_{m}{ }^{\alpha}, \\
A_{\mu}{ }^{\alpha} & \equiv \hat{A}_{\mu}{ }^{\alpha}-A_{\mu}^{(1) m} \hat{A}_{m}{ }^{\alpha} .
\end{aligned}
$$

Solving for the hatted components we get

$$
\begin{aligned}
& \hat{A}_{m}{ }^{\alpha}=a_{m}{ }^{\alpha}, \\
& \hat{A}_{\mu}{ }^{\alpha}=A_{\mu}{ }^{\alpha}+A_{\mu}^{(1) m} a_{m}{ }^{\alpha} .
\end{aligned}
$$

From the two-form potentials $\hat{b}_{\hat{\mu} \hat{\nu}}$ we get scalar fields $B_{m n}$, lower-dimensional abelian gauge fields $A_{\mu m}^{(2)}$, and a lower-dimensional two-form $b_{\mu \nu}$ defined from the relations: ${ }^{7}$

$$
\begin{aligned}
B_{m n} & \equiv \hat{b}_{m n}, \\
A_{\mu m}^{(2)} & \equiv \hat{b}_{\mu m}-A_{\mu}^{(1) k} \hat{b}_{k m}+\frac{1}{2} a_{m}{ }^{\alpha} A_{\mu \alpha}, \\
b_{\mu \nu} & \equiv \hat{b}_{\mu \nu}+A_{[\mu}^{(1) m} \hat{b}_{\nu] m}-\frac{1}{2} a_{m}{ }^{\alpha} A_{[\mu}^{(1) m} A_{\nu] \alpha} .
\end{aligned}
$$

Solving for the hatted components we find:

$$
\begin{aligned}
& \hat{b}_{m n}=B_{m n}, \\
& \hat{b}_{\mu m}=A_{\mu m}^{(2)}-A_{\mu}^{(1) n} B_{m n}-\frac{1}{2} a_{m}{ }^{\alpha} A_{\mu \alpha}, \\
& \hat{b}_{\mu \nu}=b_{\mu \nu}-A_{[\mu}^{(1) m} A_{\nu] m}^{(2)}+A_{\mu}^{(1) m} A_{\nu}^{(1) n} B_{m n}+a_{m}{ }^{\alpha} A_{[\mu}^{(1) m} A_{\nu] \alpha} .
\end{aligned}
$$

\footnotetext{
${ }^{7}$ The last terms on the right hand side of the last two equations in (3.25) are needed due to the presence of the Chern-Simons term in the $D$ dimensional action. Also note that, apart from those terms, the definition of $b_{\mu \nu}$ in terms of $\hat{b}_{\hat{\mu} \hat{\nu}}$ differs from the prescription given earlier: the second term on the right hand side has coefficient 1 rather than 2 and there is a missing $A^{(1)} A^{(1)} \hat{b}$ term. As a result, $b_{\mu \nu}$ has an anomalous transformation under the gauge symmetry associated with $A_{\mu}^{(1) m}$. However, it also has an anomalous transformation under the gauge symmetry associated with $A_{\mu}^{(2)}{ }_{m}$, and only for the above redefinition do they combine into a manifestly $O(d, d)$ invariant gauge symmetry.
} 
Note that the abelian gauge fields arising from the metric have the internal index up while those arising from the antisymmetric two-form have the internal index down. The superscripts (1) and (2) are thus not strictly needed, but they help distinguish those two sets. For these gauge fields we neither raise nor lower the internal index. A straightforward but somewhat tedious computation gives for the field strength in terms of the redefined fields,

$$
\begin{aligned}
H_{m n k} & =-f_{\alpha \beta \gamma} a_{m}{ }^{\alpha} a_{n}{ }^{\beta} a_{k}{ }^{\gamma}, \\
H_{\mu m n} & =\partial_{\mu} B_{m n}+a_{[m}{ }^{\alpha} D_{\mu} a_{n] \alpha}, \\
H_{\mu \nu m} & =\mathcal{F}_{\mu \nu m}^{(2)}-C_{m n} \mathcal{F}_{\mu \nu}^{(1) n}-a_{m}{ }^{\alpha} \mathcal{F}_{\mu \nu \alpha},
\end{aligned}
$$

where we use the covariant derivatives, non-abelian field strengths, abelian field strengths, and the auxiliary scalars defined by

$$
\begin{aligned}
D_{\mu} a_{m \alpha} & \equiv \partial_{\mu} a_{m \alpha}-A_{\mu}^{\gamma} f_{\gamma \alpha \beta} a_{m}{ }^{\beta}, \\
\mathcal{F}_{\mu \nu \alpha} & \equiv 2 \partial_{[\mu} A_{\nu] \alpha}+f_{\alpha \beta \gamma} A_{\mu}{ }^{\beta} A_{\nu}{ }^{\gamma}, \\
\mathcal{F}_{\mu \nu}^{(1) m} & \equiv \partial_{\mu} A_{\nu}^{(1) m}-\partial_{\nu} A_{\mu}^{(1) m}, \\
\mathcal{F}_{\mu \nu m}^{(2)} & \equiv \partial_{\mu} A_{\nu m}^{(2)}-\partial_{\nu} A_{\mu m}^{(2)}, \\
C_{m n} & \equiv B_{m n}+\frac{1}{2} a_{m}{ }^{\alpha} a_{n \alpha} .
\end{aligned}
$$

The most laborious part of the calculation is to verify that

$$
H_{\mu \nu \rho}=3\left(\partial_{[\mu} b_{\nu \rho]}-A_{[\mu}^{(1) m} \partial_{\nu} A_{\rho] m}^{(2)}-\partial_{[\nu} A_{\rho}^{(1) m} A_{\mu] m}^{(2)}-A_{[\mu}^{\alpha} \partial_{\nu} A_{\rho] \alpha}-\frac{1}{3} f_{\alpha \beta \gamma} A_{\mu}{ }^{\alpha} A_{\nu}{ }^{\beta} A_{\rho}^{\gamma}\right),
$$

so that it can be combined into the $O(d, d)$ covariant form, to be given in (5.46) below. Similarly, the components of the (redefined) Yang-Mills field strength become

$$
\begin{aligned}
F_{\mu \nu}{ }^{\alpha} & =\mathcal{F}_{\mu \nu}{ }^{\alpha}+\mathcal{F}_{\mu \nu}^{(1) m} a_{m}{ }^{\alpha}, \\
F_{\mu m}{ }^{\alpha} & =D_{\mu} a_{m}{ }^{\alpha}, \\
F_{m n}{ }^{\alpha} & =f^{\alpha}{ }_{\beta \gamma} a_{m}{ }^{\beta} a_{n}{ }^{\gamma} .
\end{aligned}
$$

This is close to the Maharana-Schwarz result but there are some differences. First, all partial derivatives become covariant derivatives when acting on objects with $\alpha$ index. Second, abelian field strengths become non-abelian field strengths. Finally, we have additional terms involving purely internal field strength components.

Given (3.22) and the above results, the last contribution can be expressed as

$$
-V=-\frac{1}{12} f_{\alpha \beta \gamma} f_{\alpha^{\prime} \beta^{\prime} \gamma^{\prime}} a_{m}{ }^{\alpha} a_{n}{ }^{\beta} a_{k}{ }^{\gamma} a^{m \alpha^{\prime}} a^{n \beta^{\prime}} a^{k \gamma^{\prime}}-\frac{1}{4} f_{\beta \gamma}^{\alpha} f_{\alpha \beta^{\prime} \gamma^{\prime}} a_{m}{ }^{\beta} a_{n}{ }^{\gamma} a^{m \beta^{\prime}} a^{n \gamma^{\prime}} .
$$

We summarize this section by assembling the pieces and giving the final form of the dimensionally reduced action:

$$
\begin{aligned}
S=\int d^{n} x \sqrt{-g} e^{-2 \phi}[ & R(g)+4 \partial^{\mu} \phi \partial_{\mu} \phi+\frac{1}{4} \partial^{\mu} G^{m n} \partial_{\mu} G_{m n}-\frac{1}{4} G_{m n} \mathcal{F}^{\mu \nu(1) m} \mathcal{F}_{\mu \nu}^{(1) n} \\
& -\frac{1}{4} \kappa_{\alpha \beta} F^{\mu \nu \alpha} F_{\mu \nu}{ }^{\beta}-\frac{1}{2} \kappa_{\alpha \beta} G_{m n} F^{\mu m \alpha} F_{\mu}{ }^{n \beta} \\
& \left.-\frac{1}{12} H^{\mu \nu \rho} H_{\mu \nu \rho}-\frac{1}{4} H^{\mu \nu m} H_{\mu \nu m}-\frac{1}{4} H^{\mu m n} H_{\mu m n}-V\right]
\end{aligned}
$$


Here the volume element takes the 'string frame' canonical form thanks to the redefinition

$$
\phi=\widehat{\phi}-\frac{1}{4} \log \operatorname{det} G_{m n} .
$$

The terms in the first line of the action originate from the Einstein-Hilbert and dilaton terms in $D$ dimensions, which are not affected by the non-abelian gauge couplings and therefore can be taken directly from [7]. The terms in the second line originate from the Yang-Mills kinetic term, cf. (3.20), while the terms in the third line other than $V$ originate from the kinetic term of the $b$-field in (3.21). Finally, the potential $V$ is given in (3.31) and encodes the terms not present in the Maharana-Schwarz analysis (beyond those originating from covariantizing gauge couplings).

In the next section we write the above action in an $O(d, d+K)$ covariant form, with $K=\operatorname{dim} G$, although the theory only has $O(d, d)$ as a proper symmetry. For this purpose we assemble the terms in the above action in slightly different order

$$
\begin{aligned}
S=\int d^{n} x \sqrt{-g} e^{-2 \phi}[ & R(g)+4 \partial^{\mu} \phi \partial_{\mu} \phi-\frac{1}{12} H^{\mu \nu \rho} H_{\mu \nu \rho} \\
& +\frac{1}{4} \partial^{\mu} G^{m n} \partial_{\mu} G_{m n}-\frac{1}{2} \kappa_{\alpha \beta} G_{m n} F^{\mu m \alpha} F_{\mu}{ }^{n \beta}-\frac{1}{4} H^{\mu m n} H_{\mu m n} \\
& \left.-\frac{1}{4} G_{m n} \mathcal{F}^{\mu \nu(1) m} \mathcal{F}_{\mu \nu}^{(1) n}-\frac{1}{4} \kappa_{\alpha \beta} F^{\mu \nu \alpha} F_{\mu \nu}{ }^{\beta}-\frac{1}{4} H^{\mu \nu m} H_{\mu \nu m}-V\right] .
\end{aligned}
$$

\section{Compactified theory in terms of $O(d, d+K)$ multiplets}

We now rewrite the action (3.34) in a form that is covariant under $O(d, d+K)$, where $K$ is the dimension of the gauge algebra. The gauge algebra type will be discussed below. We will use the convention that indices and objects transforming covariantly under $O(d, d+K)$ are hatted. This should not be confused with the use of hats in the previous section, where they refer to higher-dimensional objects and indices. Furthermore from this section onwards we shall use the symbol $\hat{\eta}$ and $\eta$ to describe respectively the $O(d, d+K)$ and $O(d, d)$ invariant metric and not the Minkowski metric as in the last section. We now claim that the dimensionally reduced action (3.34) can be written $\mathrm{as}^{8}$

$$
\begin{aligned}
S=\int d^{n} x \sqrt{-g} e^{-2 \phi} & \left(R(g)+4 \partial^{\mu} \phi \partial_{\mu} \phi-\frac{1}{12} H^{\mu \nu \rho} H_{\mu \nu \rho}\right. \\
& \left.+\frac{1}{8} D^{\mu} \widehat{\mathcal{H}}^{\hat{M} \hat{N}} D_{\mu} \widehat{\mathcal{H}}_{\hat{M} \hat{N}}-\frac{1}{4} \widehat{\mathcal{H}}_{\hat{M} \hat{N}} \widehat{\mathcal{F}}^{\mu \nu \hat{M}} \widehat{\mathcal{F}}_{\mu \nu} \hat{N}-V(\widehat{\mathcal{H}})\right),
\end{aligned}
$$

where

$$
\begin{aligned}
D_{\mu} \widehat{\mathcal{H}}^{\hat{M} \hat{N}} & \equiv \partial_{\mu} \widehat{\mathcal{H}}^{\hat{M} \hat{N}}-2 \widehat{A}_{\mu} \hat{K}_{\hat{K}}\left(\hat{M}{ }_{\hat{L}} \widehat{\mathcal{H}}^{\hat{N}) \hat{L}}\right. \\
\widehat{\mathcal{F}}_{\mu \nu} \hat{M} & \equiv \partial_{\mu} \widehat{A}_{\nu}^{\hat{M}}-\partial_{\nu} \widehat{A}_{\mu}^{\hat{M}}+f^{\hat{M}} \hat{K} \hat{L}_{\mu} \widehat{A}_{\mu} \widehat{A}_{\nu} \hat{L} \\
H_{\mu \nu \rho} & \equiv 3\left(\partial_{[\mu} b_{\nu \rho]}-\widehat{A}_{[\mu}^{\hat{M}} \partial_{\nu} \widehat{A}_{\rho] \hat{M}}-\frac{1}{3} f_{\hat{M} \hat{K} \hat{L}} \widehat{A}_{[\mu} \hat{M} \widehat{A}_{\nu} \hat{K}^{\hat{A}} \widehat{A}_{\rho]} \hat{L}\right),
\end{aligned}
$$

\footnotetext{
${ }^{8}$ This action is of the same structural form as that obtained by Scherk-Schwarz compactification of heterotic supergravity truncated to the Cartan subalgebra [30]. Moreover, it is closely related to that given in [31], which considers group manifold reductions of heterotic supergravity including non-abelian gauge fields and also displays the action with a formal $O(d, d+K)$ symmetry.
} 
and the potential is

$$
V(\mathcal{H})=\frac{1}{12} f^{\hat{M}}{ }_{\hat{K} \hat{P}} f^{\hat{N}}{ }_{\hat{L} \hat{Q}} \widehat{\mathcal{H}}_{\hat{M} \hat{N}} \widehat{\mathcal{H}}^{\hat{K} \hat{L}} \widehat{\mathcal{H}}^{\hat{P} \hat{Q}}+\frac{1}{4} f^{\hat{M}} \hat{N} \hat{K} f^{\hat{N}}{ }_{\hat{M} \hat{L}} \widehat{\mathcal{H}}^{\hat{K} \hat{L}}+\frac{1}{6} f^{\hat{M} \hat{N} \hat{K}} f_{\hat{M} \hat{N} \hat{K}}
$$

Here the $f^{\hat{M}} \hat{N} \hat{K}$ are a set of constants which we shall call structure constants, and the indices take values $\hat{M}, \hat{N}, \ldots=1, \ldots, 2 d+K$. These indices are lowered and raised with a metric $\widehat{\eta}_{\hat{M} \hat{N}}$ and its inverse $\widehat{\eta}^{\hat{M} \hat{N}} \equiv\left(\widehat{\eta}^{-1}\right)^{\hat{M} \hat{N}}$ :

$$
\widehat{\eta}_{\hat{M} \hat{N}} \equiv\left(\begin{array}{ccc}
0 & \delta^{i}{ }_{j} & 0 \\
\delta_{i}{ }^{j} & 0 & 0 \\
0 & 0 & \kappa_{\alpha \beta}
\end{array}\right) .
$$

Since $\kappa$ is invertible, $\widehat{\eta}$ is also invertible. Associated with the constant invertible metric $\widehat{\eta}$ there is a set of matrices $\Omega$ that preserve it. With $\Omega$ carrying index structure $\Omega_{\hat{M}} \hat{N}^{\text {the }}$ matrices satisfying

$$
\Omega \widehat{\eta} \Omega^{T}=\widehat{\eta},
$$

form a group under multiplication. Because all indices are properly contracted, the action is invariant under duality transformations

$$
\begin{aligned}
\widehat{\mathcal{H}}_{\hat{M} \hat{N}} & \rightarrow\left(\Omega \widehat{\mathcal{H}} \Omega^{T}\right)_{\hat{M} \hat{N}}, \quad \widehat{A}_{\mu}^{\hat{M}} \rightarrow\left(\widehat{\eta}^{-1} \Omega \widehat{\eta}\right)^{\hat{M}} \hat{N}_{\hat{A}} \widehat{A}_{\mu}^{\hat{N}}, \\
f_{\hat{M} \hat{N} \hat{K}} & \rightarrow \Omega_{\hat{M}} \hat{M}^{\prime} \Omega_{\hat{N}} \hat{N}^{\prime} \Omega_{\hat{K}} \hat{K}^{\prime} f_{\hat{M}^{\prime} \hat{N}^{\prime} \hat{K}^{\prime}} .
\end{aligned}
$$

The action (4.1) is invariant if we set the structure constants to zero, but non-zero values of the structure constants $f$ will typically break this to a subgroup.

Let us now discuss the duality group that arises for the metric $\hat{\eta}$ in (4.4). If the matrices $\Omega$ satisfying (4.5) are changed to $A^{-1} \Omega A$, with $A$ invertible, they still form the same group. This time, however, the invariant metric is changed $\widehat{\eta} \rightarrow A \widehat{\eta} A^{T}$. If the Lie algebra of the theory is compact and semisimple the Cartan-Killing metric $\kappa$ is positive definite and there is a matrix $\omega$ such that $\omega \kappa \omega^{T}=I_{K}$, with $I_{K}$ the $K \times K$ identity matrix. It then follows that by taking $A$ to be of the block-diagonal form $\left(I_{d}, \omega\right)$, the metric $\widehat{\eta}$ can be put in the form $\left(\eta, I_{K}\right)$, with $\eta$ the $O(d, d)$ metric. We then recognize that for compact semisimple Lie algebras we have the duality group $O(d, d+K)$. The case when the gauge group contains $\mathrm{U}(1)$ factors will be discussed at the end of the section. These are the situations we have in mind, and we will simply speak of $O(d, d+K)$ as the duality group. We have introduced explicitly the Cartan-Killing metric, however, to allow for the possibility of future generalizations, including non-compact semisimple algebras, as we discuss in the conclusions.

In order to make contact with (3.34) we need the explicit expressions for $\widehat{\mathcal{H}}_{\hat{M} \hat{N}}$ and $\widehat{A}_{\mu} \hat{M}$ in terms of the fields obtained in the previous section after dimensional reduction, and also the values of the structure constants $f_{\hat{M} \hat{N} \hat{K}}$. The matrix $\widehat{\mathcal{H}}$ is parameterized by the internal (scalar) components $G, B$, and $a$ of the metric, $b$-field, and gauge fields, 
respectively as follows:

$$
\begin{aligned}
\widehat{\mathcal{H}}_{\hat{M} \hat{N}} & =\left(\begin{array}{ccc}
\widehat{\mathcal{H}}^{m n} & \widehat{\mathcal{H}}^{m}{ }_{n} & \widehat{\mathcal{H}}^{m} \\
\widehat{\mathcal{H}}_{m}{ }^{n} & \widehat{\mathcal{H}}_{m n} & \widehat{\mathcal{H}}_{m \beta} \\
\widehat{\mathcal{H}}_{\alpha}{ }^{n} & \widehat{\mathcal{H}}_{\alpha n} & \widehat{\mathcal{H}}_{\alpha \beta}
\end{array}\right) \\
& =\left(\begin{array}{ccc}
G^{m n} & -G^{m k} C_{k n} & -G^{m k} a_{k \beta} \\
-G^{n k} C_{k m} & G_{m n}+C_{k m} G^{k l} C_{l n}+a_{m}^{\gamma} a_{n \gamma} & C_{k m} G^{k l} a_{l \beta}+a_{m \beta} \\
-G^{n k} a_{k \alpha} & C_{k n} G^{k l} a_{l \alpha}+a_{n \alpha} & \kappa_{\alpha \beta}+a_{k \alpha} G^{k l} a_{l \beta}
\end{array}\right),
\end{aligned}
$$

with

$$
C_{m n}=B_{m n}+\frac{1}{2} a_{m}{ }^{\alpha} a_{n \alpha} .
$$

It is easy to see that the generalized metric $\widehat{\mathcal{H}}$ with matrix element $\widehat{\mathcal{H}}_{\hat{M} \hat{N}}$ satisfies:

$$
\widehat{\mathcal{H}} \widehat{\eta}^{-1} \in O(d, d+K) \Longleftrightarrow \widehat{\mathcal{H}}_{\hat{M}} \hat{P}^{\widehat{\mathcal{H}}_{\hat{N}}} \hat{Q}_{\hat{\eta}} \hat{Q}=\widehat{\eta}_{\hat{M} \hat{N}} .
$$

The gauge fields $A_{\mu m}^{(2)}, A_{\mu}^{(1) m}$, and $A_{\mu}^{\alpha}$ of the previous section are combined into an $O(d, d+K)$ vector as

$$
\widehat{A}_{\mu}^{\hat{M}} \equiv\left(A_{\mu m}^{(2)}, A_{\mu}^{(1) m}, A_{\mu}^{\alpha}\right),
$$

and so are the corresponding field strengths,

$$
\widehat{\mathcal{F}}_{\mu \nu}^{\hat{M}} \equiv\left(\mathcal{F}_{\mu \nu m}^{(2)}, \mathcal{F}_{\mu \nu}^{(1) m}, \mathcal{F}_{\mu \nu}{ }^{\alpha}\right) .
$$

The first two field strengths are abelian while the final one takes the non-abelian form (3.28). Finally the structure constants are chosen to be

$$
f_{\hat{N} \hat{K}}^{\hat{M}}=\left\{\begin{array}{ll}
f^{\alpha}{ }_{\beta \gamma} & \text { if }(\hat{M}, \hat{N}, \hat{K})=(\alpha, \beta, \gamma) \\
0 & \text { otherwise }
\end{array},\right.
$$

with $\alpha, \beta$ denoting the $K$ gauge algebra directions and where $f^{\alpha}{ }_{\beta \gamma}$ are the structure constants of the gauge group $G$.

We shall now show that the action (4.1) indeed coincides with the dimensionally reduced action (3.34). The first line on each of the two actions is exactly the same. The second line on (3.34) reproduces the $\frac{1}{8} D \widehat{\mathcal{H}} D \widehat{\mathcal{H}}$ term in (4.1). Similarly, the third line on (3.34), except for the potential $V$, reproduces the $-\frac{1}{4} \widehat{\mathcal{H}} \widehat{\mathcal{F}} \widehat{\mathcal{F}}$ term in (4.1). The only difference so far with the Maharana-Schwarz analysis is the presence of covariant derivatives and non-abelian field strengths instead of partial derivatives and abelian field strengths. Since $f_{\hat{M}}^{\hat{N} \hat{K}}$ is non-trivial only in the gauge algebra directions it reproduces the nonabelian gauge structures of the reduced theory. Finally, it is a straightforward computation to verify that the potential (4.3) reproduces the potential (3.31) of the reduced theory. Inserting (4.12) into (4.3) we have

$$
V=\frac{1}{12} f_{\alpha \gamma \delta} f_{\beta \epsilon \kappa} \widehat{\mathcal{H}}^{\alpha \beta} \widehat{\mathcal{H}}^{\gamma \epsilon} \widehat{\mathcal{H}}^{\delta \kappa}+\frac{1}{4} f^{\alpha}{ }_{\beta \gamma} f^{\beta}{ }_{\alpha \delta} \widehat{\mathcal{H}}^{\gamma \delta}+\frac{1}{6} f^{\alpha \beta \gamma} f_{\alpha \beta \gamma} .
$$


This can be simplified using the value of $\widehat{\mathcal{H}}_{\alpha \beta}$ from (4.7), and the result is indeed (3.31). We finally note that in the limit $f_{\alpha \beta \gamma} \rightarrow 0$ the action reduces to that found by MaharanaSchwarz, in which case the theory is properly invariant under a global $O(d, d+K)$ symmetry.

If we are willing to accept the Maharana-Schwarz action as a valid starting point, we could arrive at the action (4.1) using the following short argument. First of all we note that the $D$ dimensional action has terms quadratic in $f_{\alpha \beta \gamma}$, linear in $f_{\alpha \beta \gamma}$ and independent of $f_{\alpha \beta \gamma}$. The MS action corresponds to terms independent of $f_{\alpha \beta \gamma}$, and as pointed out above, the $f_{\alpha \beta \gamma}$ independent part of the action (4.1) coincides with the MS action. Thus we only need to verify that the terms linear and quadratic in $f_{\alpha \beta \gamma}$ are correct. Now by examining the $D$ dimensional action (3.2) we see that all the terms linear in $f_{\alpha \beta \gamma}$ have a single derivative and all the terms quadratic in $f_{\alpha \beta \gamma}$ have no derivatives. Thus the dimensionally reduced action must also have this property. We see that (4.1) does share this property. Thus if (4.1) is not the correct dimensionally reduced action then any additional term must share this property. Furthermore since both the original action (3.2) and the dimensionally reduced action (4.1) are gauge invariant, any additional term must also be gauge invariant. It is easy to see that it is impossible to write down a gauge invariant term with a single derivative involving the fields which appear in (4.1) or equivalently in (3.34). This shows that there are no additional terms with a single power of $f_{\alpha \beta \gamma}$. This leaves us to check that (4.1) reproduces correctly the derivative free terms quadratic in $f_{\alpha \beta \gamma}$, i.e. that the potential term (4.3) is correct. ${ }^{9}$ As discussed earlier, this term comes from (3.22) and can be easily computed, leading to (4.3). This shows that the action (4.1) is the correct dimensionally reduced action.

The action (4.1) given at the beginning of this section applies with some modifications when the gauge group is $G^{\prime} \times \mathrm{U}(1)^{p}$, with $G^{\prime}$ semisimple. As explained before, the $\kappa$ matrix then takes the block-diagonal form in (3.5), and the $\widehat{\eta}$ metric in (4.4) now becomes

$$
\text { For } G^{\prime} \times \mathrm{U}(1)^{p}: \quad \widehat{\eta} \equiv\left(\begin{array}{cccc}
0 & I_{d} & 0 & 0 \\
I_{d} & 0 & 0 & 0 \\
0 & 0 & I_{p} & 0 \\
0 & 0 & 0 & \kappa^{\prime}
\end{array}\right)
$$

With $G^{\prime}$ compact semisimple, this metric is associated with the duality group $O(d, d+p+$ $K^{\prime}$ ), where $K^{\prime}$ is the dimension of $G^{\prime}$. The indices now run as $\hat{M}, \hat{N} \ldots=1, \ldots, 2 d+p+K^{\prime}$. The gauge fields are now an $O\left(d, d+p+K^{\prime}\right)$ vector:

$$
\widehat{A}_{\mu}^{\hat{M}} \equiv\left(A_{\mu m}^{(2)}, A_{\mu}^{(1) m}, A_{\mu}^{i}, A_{\mu}^{\alpha^{\prime}}\right)
$$

where $A_{\mu}{ }^{i}$, with $i=1, \ldots, p$ are $p$ abelian gauge fields, and $\alpha^{\prime}=1, \ldots, K^{\prime}$. The structure constants $f^{\hat{M}} \hat{N} \hat{K}$ vanish except when all indices take values on the $K^{\prime}$ components associated with the Lie algebra of $G^{\prime}$. This time $\widehat{\mathcal{H}}$ is a $\left(2 d+p+K^{\prime}\right) \times\left(2 d+p+K^{\prime}\right)$ matrix and

$$
\widehat{\mathcal{H}} \widehat{\eta}^{-1} \in O\left(d, d+p+K^{\prime}\right) .
$$

\footnotetext{
${ }^{9}$ The form of the potential can also be read off from the $f$-dependent terms in the heterotic double field theory action given in [15].
} 
The parameterization of $\widehat{\mathcal{H}}$ can be obtained from that in (4.7) by letting the Lie algebra gauge indices run over two kinds of values: $\alpha=\left(i, \alpha^{\prime}\right)$, again, with $i=1, \ldots, p$, and $\alpha^{\prime}=$ $1, \ldots, K^{\prime}$. Moreover, we take $\kappa_{i j}=\delta_{i j}, \kappa_{\alpha^{\prime} i}=\kappa_{i \alpha^{\prime}}=0$, and $\kappa_{\alpha^{\prime} \beta^{\prime}}$ the matrix elements of $\kappa^{\prime}$.

We have emphasized that $O(d, d+K)$ (or $O\left(d, d+p+K^{\prime}\right)$ ) are formal duality symmetries of the reduced action. Let us now discuss, following [15], the surviving global duality symmetries of the reduced action. Consider first the case where $G$ is compact semisimple and of dimension $K$. We first note that in this case the tensor $f_{\hat{M}}^{\hat{N} \hat{K}}$ in (4.12) is not $O(d, d+K)$ invariant. Since the tensor vanishes whenever an index takes any of the first $2 d$ values, it is invariant under the $O(d, d)$ subgroup that shuffles these directions while leaving the gauge algebra directions inert. ${ }^{10}$ Specifically, for the gauge groups relevant for heterotic string theory we have

$$
G=\mathrm{SO}(32) \quad \text { or } \quad E_{8} \times E_{8} \quad \rightarrow \quad \text { global duality symmetry: } O(d, d) .
$$

If the gauge group is of the form $G=G^{\prime} \times \mathrm{U}(1)^{p}$, with $G^{\prime}$ semi-simple, the tensor $f^{\hat{M}} \hat{N} \hat{K}$ vanishes whenever an index takes any of the first $2 d+p$ values. Consequently, it is invariant under the larger group $O(d, d+p)$, which is the true duality symmetry. For instance, if we truncate the heterotic theory gauge group down to $E_{8} \times U(1)^{8}$, the massless effective field theory on $T^{d}$ will have:

$$
G=E_{8} \times U(1)^{8} \quad \rightarrow \quad \text { global duality symmetry: } O(d, d+8)
$$

\section{Compactified theory in terms of $O(d, d)$ multiplets}

In the previous sections we have considered the heterotic string with its full non-abelian gauge group $G$ compactified on a torus. The low-energy effective field theory action was displayed with a formal $O(d, d+K)$ global symmetry, with $K$ the dimension of the nonabelian gauge group. We have also seen that the true global symmetry of the low energy effective action is $O(d, d) \times G$ for compactifications without Wilson lines, and the gauge fields give rise to massless adjoint scalars and lower-dimensional massless gauge fields of $G$. The purpose of this section is to make this symmetry manifest by rewriting the low-energy action (4.1) in terms of proper $O(d, d) \times G$ multiplets, instead of the fictitious $O(d, d+K)$ multiplets. The fields that will be used are

$$
\mathcal{H}_{M N}, \quad \mathcal{C}_{M}{ }^{\alpha}, \quad \text { with constraints: } \quad \mathcal{H} \eta \mathcal{H}=\eta, \quad(1+\mathcal{H} \eta) \mathcal{C}=0
$$

When some Wilson lines are included in the heterotic compactification, the gauge group $G$ can be broken to a group $G^{\prime} \times \mathrm{U}(1)^{p}$. The duality group of the low-energy effective theory for the massless fields is enhanced to $O(d, d+p)$. The analysis of this section can also be generalized to make the $O(d, d+p)$ symmetry of the action manifest by using $O(d, d+p) \times G^{\prime}$ multiplets.

\footnotetext{
${ }^{10}$ More precisely, the global subgroup leaving (4.12) invariant is $O(d, d) \times G$, with $G$ the rigid subgroup of the gauge group.
} 


\subsection{Introducing $O(d, d)$ field multiplets}

Let us consider the $(2 d+K) \times(2 d+K)$ generalized metric of equation (4.7) written in block form as follows:

$$
\widehat{\mathcal{H}}=\left(\begin{array}{cc}
\widetilde{\mathcal{H}} & \widetilde{\mathcal{C}} \\
\widetilde{\mathcal{C}}^{T} & \widetilde{\mathcal{N}}
\end{array}\right)
$$

With more explicit index notation

$$
\widehat{\mathcal{H}}_{\hat{M} \hat{N}}=\left(\begin{array}{cc}
\widetilde{\mathcal{H}}_{M N} & \widetilde{\mathcal{C}}_{M \beta} \\
\left(\widetilde{\mathcal{C}}^{T}\right)_{\alpha N} & \widetilde{\mathcal{N}}_{\alpha \beta}
\end{array}\right),
$$

where now the indices $M, N$ run over $2 d$ values. Thus the matrix dimensions are as follows

$$
\begin{aligned}
\widehat{\eta}, \widehat{\mathcal{H}} & :(2 d+K) \times(2 d+K), \\
\eta, \widetilde{\mathcal{H}} & :(2 d) \times(2 d), \\
\widetilde{\mathcal{C}} & :(2 d) \times K, \\
\kappa, \widetilde{\mathcal{N}} & : K \times K .
\end{aligned}
$$

Since $\widehat{\mathcal{H}} \widehat{\eta}^{-1}$ is an $O(d, d+K)$ matrix it satisfies $\widehat{\mathcal{H}} \widehat{\eta}^{-1} \widehat{\mathcal{H}}=\widehat{\eta}$ (see (4.9)) and therefore

$$
\left(\begin{array}{cc}
\widetilde{\mathcal{H}} & \widetilde{\mathcal{C}} \\
\widetilde{\mathcal{C}}^{T} & \widetilde{\mathcal{N}}
\end{array}\right)\left(\begin{array}{cc}
\eta & 0 \\
0 & \kappa^{-1}
\end{array}\right)\left(\begin{array}{cc}
\widetilde{\mathcal{H}} & \widetilde{\mathcal{C}} \\
\widetilde{\mathcal{C}}^{T} & \widetilde{\mathcal{N}}
\end{array}\right)=\left(\begin{array}{ll}
\eta & 0 \\
0 & \kappa
\end{array}\right),
$$

where

$$
\eta \equiv\left(\begin{array}{cc}
0 & \delta^{i}{ }_{j} \\
\delta_{i}{ }^{j} & 0
\end{array}\right)
$$

is the $O(d, d)$ invariant tensor. The equality (5.5) implies three conditions for the block matrices:

$$
\begin{aligned}
\widetilde{\mathcal{H}} \eta \widetilde{\mathcal{H}}+\widetilde{\mathcal{C}} \kappa^{-1} \widetilde{\mathcal{C}}^{T} & =\eta, \\
\widetilde{\mathcal{H}} \eta \widetilde{\mathcal{C}}+\widetilde{\mathcal{C}} \kappa^{-1} \widetilde{\mathcal{N}} & =0, \\
\widetilde{\mathcal{C}}^{T} \eta \widetilde{\mathcal{C}}+\widetilde{\mathcal{N}} \kappa^{-1} \widetilde{\mathcal{N}} & =\kappa .
\end{aligned}
$$

We shall now try to find a suitable parametrization of $\widetilde{\mathcal{H}}, \widetilde{\mathcal{C}}$, and $\widetilde{\mathcal{N}}$ satisfying these relations. First of all, the last condition in (5.7) shows that $\widetilde{\mathcal{N}}$ and $\widetilde{\mathcal{C}}$ are not independent variables. A useful way to express this dependence is to introduce a new $O(d, d)$ vector $\mathcal{C}$ via the equation

$$
\widetilde{\mathcal{C}}=\mathcal{C}\left(1+\kappa^{-1} \tilde{\mathcal{N}}\right)
$$

Indeed, the equation leads to

$$
\left(1+\widetilde{\mathcal{N}} \kappa^{-1}\right) \mathcal{C}^{T} \eta \mathcal{C}\left(1+\kappa^{-1} \tilde{\mathcal{N}}\right)=\kappa-\tilde{\mathcal{N}} \kappa^{-1} \widetilde{\mathcal{N}}=\kappa\left(1-\kappa^{-1} \widetilde{\mathcal{N}}\right)\left(1+\kappa^{-1} \tilde{\mathcal{N}}\right)
$$

giving us

$$
\widetilde{\mathcal{N}}=\left(\kappa-\mathcal{C}^{T} \eta \mathcal{C}\right)\left(\kappa+\mathcal{C}^{T} \eta \mathcal{C}\right)^{-1} \kappa=\kappa\left(\kappa+\mathcal{C}^{T} \eta \mathcal{C}\right)^{-1}\left(\kappa-\mathcal{C}^{T} \eta \mathcal{C}\right)
$$


Eqs. (5.10) and (5.8) express both $\widetilde{\mathcal{N}}$ and $\widetilde{\mathcal{C}}$ in terms of $\mathcal{C}$. For later use we note that

$$
\kappa^{-1}+\kappa^{-1} \widetilde{\mathcal{N}} \kappa^{-1}=\kappa^{-1}\left[\left(\kappa+\mathcal{C}^{T} \eta \mathcal{C}\right)+\left(\kappa-\mathcal{C}^{T} \eta \mathcal{C}\right)\right]\left(\kappa+\mathcal{C}^{T} \eta \mathcal{C}\right)^{-1}=2\left(\kappa+\mathcal{C}^{T} \eta \mathcal{C}\right)^{-1}
$$

We now introduce an $O(d, d)$ valued generalized metric and an $O(d, d)$ vector to parametrize the above fields. We claim that the first two conditions in (5.7) can be solved by taking

$$
\widetilde{\mathcal{H}}=\mathcal{H}+\mathcal{C}\left(\kappa^{-1}+\kappa^{-1} \widetilde{\mathcal{N}} \kappa^{-1}\right) \mathcal{C}^{T}
$$

where $\mathcal{H}$ is a symmetric matrix satisfying: ${ }^{11}$

$$
\mathcal{H} \eta \mathcal{H}=\eta, \quad(1+\mathcal{H} \eta) \mathcal{C}=0
$$

This can be easily verifed by substituting (5.12) into the first two equations of (5.7) and using (5.13), (5.8), and (5.10). Furthermore since $\mathcal{H}$ is determined uniquely from $\widetilde{\mathcal{H}}, \widetilde{\mathcal{C}}$, and $\widetilde{\mathcal{N}}$ using eqs. (5.12) and (5.8), (5.12) is the most general form of $\widetilde{\mathcal{H}}$ satisfying (5.7).

These results can now by summarized in the statement that $\widetilde{\mathcal{H}}, \widetilde{\mathcal{C}}$, and $\widetilde{\mathcal{N}}$ satisfying $(5.7)$ can be parametrized by $\mathcal{H}$ and $\mathcal{C}$ satisfying (5.13) via the relations

$$
\begin{aligned}
\widetilde{\mathcal{H}} & =\mathcal{H}+\mathcal{C}\left(\kappa^{-1}+\kappa^{-1} \widetilde{\mathcal{N}} \kappa^{-1}\right) \mathcal{C}^{T}, \\
\widetilde{\mathcal{C}} & =\mathcal{C}\left(1+\kappa^{-1} \widetilde{\mathcal{N}}\right) \\
1+\widetilde{\mathcal{N}} \kappa^{-1} & =2 \kappa\left(\kappa+\mathcal{C}^{T} \eta \mathcal{C}\right)^{-1}
\end{aligned}
$$

Alternatively, using (5.11), this can be written as

$$
\begin{aligned}
\widetilde{\mathcal{H}} & =\mathcal{H}+2 \mathcal{C}\left(\kappa+\mathcal{C}^{T} \eta \mathcal{C}\right)^{-1} \mathcal{C}^{T}, \\
\widetilde{\mathcal{C}} & =2 \mathcal{C}\left(\kappa+\mathcal{C}^{T} \eta \mathcal{C}\right)^{-1} \kappa, \\
\widetilde{\mathcal{N}} & =-\kappa+2 \kappa\left(\kappa+\mathcal{C}^{T} \eta \mathcal{C}\right)^{-1} \kappa .
\end{aligned}
$$

Our next goal is to write the proper $O(d, d)$ covariant objects $\mathcal{H}$ and $\mathcal{C}$ in terms of the physical fields. ${ }^{12}$ Thus, consider the expressions for $\widetilde{\mathcal{H}}, \widetilde{\mathcal{C}}$, and $\widetilde{\mathcal{N}}$ in terms of the dimensionally reduced physical variables given in (4.7). Using matrix notation for $G, B$,

\footnotetext{
${ }^{11}$ The second condition is a projector condition because $P \equiv \frac{1}{2}(1+\mathcal{H} \eta)$ satisfies $P^{2}=P$. The complementary orthogonal projector is $\bar{P} \equiv \frac{1}{2}(1-\mathcal{H} \eta)$. We now explain that the alternative condition $(1-\mathcal{H} \eta) \mathcal{C}=0$ would not be viable. Consider the second constraint in (5.7), $\widetilde{\mathcal{H}} \eta \widetilde{\mathcal{C}}+\widetilde{\mathcal{C}} \kappa^{-1} \widetilde{\mathcal{N}}=0$, perturbatively around zero $\mathcal{C}$. To leading order we have $\widetilde{\mathcal{N}}=\kappa$ and we require $\widetilde{\mathcal{C}}=\mathcal{C}$ and $\widetilde{\mathcal{H}}=\mathcal{H}$. The constraint becomes $(\mathcal{H} \eta+1) \mathcal{C}=0$. The choice of this projector was fixed by our convention for the duality group and its associated metric. We picked $O(d, d+K)$ where $d+K$ is the number of positive eigenvalues of $\widehat{\eta}$, including the positive eigenvalues of $\kappa$. Had we chosen $O(d+K, d)$, we would have to change $\kappa \rightarrow-\kappa$ in $\widehat{\eta}$ and the other projector would have been selected.

${ }^{12}$ Ref. [29] has examined the use of unconstrained $O(d, d)$ vectors for a DFT of Yang-Mills fields.
} 
and $(a)^{\alpha}{ }_{i} \equiv a_{i}{ }^{\alpha}$ we read off

$$
\begin{aligned}
& \widetilde{\mathcal{H}}=\left(\begin{array}{cc}
G^{-1} & -G^{-1}\left(B+\frac{1}{2} a^{T} \kappa a\right) \\
\left(B-\frac{1}{2} a^{T} \kappa a\right) G^{-1} & G+\left(-B+\frac{1}{2} a^{T} \kappa a\right) G^{-1}\left(B+\frac{1}{2} a^{T} \kappa a\right)+a^{T} \kappa a
\end{array}\right) \\
& \widetilde{\mathcal{C}}=\left(\begin{array}{c}
-G^{-1} a^{T} \kappa \\
\left(-B+\frac{1}{2} a^{T} \kappa a\right) G^{-1} a^{T} \kappa+a^{T} \kappa
\end{array}\right)=\left(\begin{array}{c}
-G^{-1} a^{T} \kappa \\
(-B+\bar{G}) G^{-1} a^{T} \kappa
\end{array}\right) \\
& \widetilde{\mathcal{N}}=\kappa+\kappa a G^{-1} a^{T} \kappa .
\end{aligned}
$$

Here we defined

$$
\bar{G} \equiv G+\frac{1}{2} a^{T} \kappa a,
$$

for later convenience. Using this we can now express the new field variables $\mathcal{H}$ and $\mathcal{C}$ in terms of the physical fields. We begin with $\mathcal{C}$ :

$$
\mathcal{C}=\widetilde{\mathcal{C}}\left(1+\kappa^{-1} \widetilde{\mathcal{N}}\right)^{-1}=\left(\begin{array}{c}
-G^{-1} a^{T} \kappa \\
(-B+\bar{G}) G^{-1} a^{T} \kappa
\end{array}\right) \frac{1}{2}\left(1+\frac{1}{2} a G^{-1} a^{T} \kappa\right)^{-1} .
$$

Working out the geometric series one finds that this can be written in terms of the redefined metric $\bar{G}$ :

$$
\mathcal{C}=\frac{1}{2}\left(\begin{array}{c}
-\bar{G}^{-1} a^{T} \kappa \\
(-B+\bar{G}) \bar{G}^{-1} a^{T} \kappa
\end{array}\right)=\frac{1}{2}\left(\begin{array}{c}
-\bar{G}^{-1} a^{T} \kappa \\
-B \bar{G}^{-1} a^{T} \kappa+a^{T} \kappa
\end{array}\right) .
$$

Next we turn to $\mathcal{H}$. From (5.14) and the last of (5.16) we have

$$
\mathcal{H}=\widetilde{\mathcal{H}}-\mathcal{C}\left(\kappa^{-1}+\kappa^{-1} \widetilde{\mathcal{N}} \kappa^{-1}\right) \mathcal{C}^{T}=\widetilde{\mathcal{H}}-2 \mathcal{C}\left(\kappa^{-1}+\frac{1}{2} a G^{-1} a^{T}\right) \mathcal{C}^{T}
$$

Using our expression for $\mathcal{C}$ in terms of the physical fields and that for $\widetilde{\mathcal{H}}$, an explicit computation gives a very simple result for $\mathcal{H}$ :

$$
\mathcal{H}=\left(\begin{array}{cc}
\bar{G}^{-1} & -\bar{G}^{-1} B \\
B \bar{G}^{-1} & \bar{G}-B \bar{G}^{-1} B
\end{array}\right) .
$$

This $O(d, d)$ valued generalized metric $\mathcal{H}$ takes the usual form with the metric $G$ replaced by $\bar{G}$. It is a good exercise to verify that the constraint on $\mathcal{C}$ holds: $(1+\mathcal{H} \eta) \mathcal{C}=0$.

Since $\mathcal{H}$ transforms in the familiar way under $O(d, d)$ dualities, the fields $\bar{G}$ and $B$ transform in the familiar way. Since the transformation of $\bar{G}$ is known and $\mathcal{C}$ transforms as an $O(d, d)$ vector, this determines the duality transformation of the scalar fields $a$. Given this, one can find the duality transformations of $G$.

Assembling the $O(d, d)$ multiplets for the gauge fields requires no work. Recalling equations (4.10) and (4.11) we write now

$$
\begin{aligned}
\widehat{A}_{\mu}{ }^{\hat{M}} & \equiv\left(A_{\mu}{ }^{M}, A_{\mu}{ }^{\alpha}\right), \\
\widehat{\mathcal{F}}_{\mu \nu}{ }^{\hat{M}} & \equiv\left(\mathcal{F}_{\mu \nu}{ }^{M}, \mathcal{F}_{\mu \nu}{ }^{\alpha}\right) .
\end{aligned}
$$

The field strengths are computed in terms of the gauge fields as

$$
\begin{aligned}
\mathcal{F}_{\mu \nu}{ }^{M} & \equiv \partial_{\mu} A_{\nu}{ }^{M}-\partial_{\nu} A_{\mu}{ }^{M}, \\
\mathcal{F}_{\mu \nu}{ }^{\alpha} & \equiv 2 \partial_{[\mu} A_{\nu]}{ }^{\alpha}+f^{\alpha}{ }_{\beta \gamma} A_{\mu}{ }^{\beta} A_{\nu}{ }^{\gamma} .
\end{aligned}
$$




\subsection{Covariant action}

We now treat $\mathcal{H}$ and $\mathcal{C}$ as independent variables and formulate the action in terms of these fields. Since the action has an explicit expression in terms of $\widetilde{\mathcal{H}}, \widetilde{\mathcal{C}}$, and $\widetilde{\mathcal{N}}$, which in turn have known expressions in terms of $\mathcal{H}$ and $\mathcal{C}$, the action is guaranteed to have an explicit expression in terms of $\mathcal{H}$ and $\mathcal{C}$.

We start with the scalar kinetic terms from (4.1),

$$
L_{\text {kin }}=\frac{1}{8} D_{\mu} \widehat{\mathcal{H}}^{\hat{M} \hat{N}} D^{\mu} \widehat{\mathcal{H}}_{\hat{M} \hat{N}}=\frac{1}{8} \operatorname{tr}\left[\left(D_{\mu} \widehat{\mathcal{H}}\right) \widehat{\eta}^{-1}\left(D^{\mu} \widehat{\mathcal{H}}\right) \widehat{\eta}^{-1}\right]
$$

where $\widehat{\eta}$ is the $O(d, d+K)$ metric (4.4). Using (5.2) and expanding the blocks,

$$
L_{\text {kin }}=\frac{1}{8} \operatorname{Tr}\left(D_{\mu} \widetilde{\mathcal{H}} \eta D^{\mu} \widetilde{\mathcal{H}} \eta+2 D_{\mu} \widetilde{\mathcal{C}}^{T} \eta D^{\mu} \widetilde{\mathcal{C}} \kappa^{-1}+D_{\mu} \widetilde{\mathcal{N}} \kappa^{-1} D^{\mu} \widetilde{\mathcal{N}} \kappa^{-1}\right)
$$

Next we insert (5.14) and use (5.10) to simplify these terms. The strategy is to rewrite all terms so that only derivatives of $\mathcal{C}$ enter. To this end one uses the second constraint in (5.13) to find

$$
\mathcal{C}^{T} \eta D_{\mu} \mathcal{H}=-D_{\mu} \mathcal{C}^{T}(1+\eta \mathcal{H})
$$

which allows us to eliminate derivatives of $\mathcal{H}$ (note that $D_{\mu} \mathcal{H}=\partial_{\mu} \mathcal{H}$ ), and the third equation in (5.14) to find

$$
D_{\mu}\left(1+\widetilde{\mathcal{N}} \kappa^{-1}\right)=-2 \kappa\left(\kappa+\mathcal{C}^{T} \eta \mathcal{C}\right)^{-1}\left(D_{\mu} \mathcal{C}^{T} \eta \mathcal{C}+\mathcal{C}^{T} \eta D_{\mu} \mathcal{C}\right)\left(\kappa+\mathcal{C}^{T} \eta \mathcal{C}\right)^{-1}
$$

which allows us to eliminate derivatives of $\widetilde{\mathcal{N}}$. A direct but tedious computation then shows that (5.25) can be written in the form

$$
\begin{aligned}
L_{\mathrm{kin}}=\operatorname{Tr}[ & \frac{1}{8} \eta \partial^{\mu} \mathcal{H} \eta \partial_{\mu} \mathcal{H}-D^{\mu} \mathcal{C}\left(\kappa+\mathcal{C}^{T} \eta \mathcal{C}\right)^{-1} D_{\mu} \mathcal{C}^{T} \eta \mathcal{H} \eta \\
& \left.-D^{\mu} \mathcal{C}^{T} \eta \mathcal{C}\left(\kappa+\mathcal{C}^{T} \eta \mathcal{C}\right)^{-1} \mathcal{C}^{T} \eta D_{\mu} \mathcal{C}\left(\kappa+\mathcal{C}^{T} \eta \mathcal{C}\right)^{-1}\right]
\end{aligned}
$$

This action can be written in various equivalent forms, some of which that may be more illuminating are given in the following. Using that with the constraints (5.13) we have $\mathcal{H}^{-1}=\eta \mathcal{H} \eta$ and hence

$$
\mathcal{C}^{T} \eta \mathcal{C}=-\mathcal{C}^{T} \eta \mathcal{H} \eta \mathcal{C}=-\mathcal{C}^{T} \mathcal{H}^{-1} \mathcal{C}
$$

we can write the action, upon cycling in the trace, as

$$
\begin{aligned}
L_{\text {kin }}=\operatorname{Tr}[ & \frac{1}{8} \partial_{\mu} \mathcal{H} \partial^{\mu} \mathcal{H}^{-1}-\mathcal{H}^{-1} D_{\mu} \mathcal{C}\left(\kappa-\mathcal{C}^{T} \mathcal{H}^{-1} \mathcal{C}\right)^{-1} D^{\mu} \mathcal{C}^{T} \\
& \left.\quad-\mathcal{H}^{-1} \mathcal{C}\left(\kappa-\mathcal{C}^{T} \mathcal{H}^{-1} \mathcal{C}\right)^{-1} \mathcal{C}^{T} \mathcal{H}^{-1} D_{\mu} \mathcal{C}\left(\kappa-\mathcal{C}^{T} \mathcal{H}^{-1} \mathcal{C}\right)^{-1} D^{\mu} \mathcal{C}^{T}\right] .
\end{aligned}
$$

Next we can group the last two terms as follows

$$
L_{\mathrm{kin}}=\operatorname{Tr}\left[\frac{1}{8} \partial_{\mu} \mathcal{H} \partial^{\mu} \mathcal{H}^{-1}-\left(\mathcal{H}^{-1}+\mathcal{H}^{-1} \mathcal{C}\left(\kappa-\mathcal{C}^{T} \mathcal{H}^{-1} \mathcal{C}\right)^{-1} \mathcal{C}^{T} \mathcal{H}^{-1}\right) D_{\mu} \mathcal{C}\left(\kappa-\mathcal{C}^{T} \mathcal{H}^{-1} \mathcal{C}\right)^{-1} D^{\mu} \mathcal{C}^{T}\right]
$$


The prefactor in the second term can be simplified, as one may verify by writing out the geometric series, to obtain

$$
L_{\mathrm{kin}}=\operatorname{Tr}\left[\frac{1}{8} \partial_{\mu} \mathcal{H} \partial^{\mu} \mathcal{H}^{-1}-\left(\mathcal{H}-\mathcal{C} \kappa^{-1} \mathcal{C}^{T}\right)^{-1} D_{\mu} \mathcal{C}\left(\kappa-\mathcal{C}^{T} \mathcal{H}^{-1} \mathcal{C}\right)^{-1} D^{\mu} \mathcal{C}^{T}\right] .
$$

This form of the kinetic terms makes it clear that the variables $\mathcal{C}_{M \alpha}$ have restricted domain since the eigenvalues of $\left(\mathcal{H}-\mathcal{C} \kappa^{-1} \mathcal{C}^{T}\right)$ and $\left(\kappa-\mathcal{C}^{T} \mathcal{H}^{-1} \mathcal{C}\right)$ should never vanish, and hence, by the positivity of these eigenvalues at $\mathcal{C}=0$, must always remain positive. By writing $\mathcal{H}=A A^{T}$ and $\kappa=B^{T} B$ for some non-singular matrices $A, B$ we can translate both these conditions into positivity of the eigenvalues of

$$
\left(I_{2 d}-\overline{\mathcal{C}} \overline{\mathcal{C}}^{T}\right), \quad \overline{\mathcal{C}} \equiv A^{-1} \mathcal{C} B^{-1} .
$$

This means that the eigenvalues of $\overline{\mathcal{C}} \overline{\mathcal{C}}^{T}$ should be less than one. A particular consequence of this is that $\operatorname{Tr}\left(\overline{\mathcal{C}} \overline{\mathcal{C}}^{T}\right)<2 d$. Since the left hand side is the sum of squares of all the components of $\overline{\mathcal{C}}$, this shows that each component of $\overline{\mathcal{C}}$ has a strict upper bound. Thus, for fixed $A$ and $B$, i.e. fixed $\mathcal{H}$ and $\kappa$, each component of $\mathcal{C}$ will also have a strict upper bound. This does not impose, however, any condition on the physical fields $a_{m}{ }^{\alpha}$. To see this we note that using the constraint $(1+\mathcal{H} \eta) \mathcal{C}=0$, the third equation in (5.14) and the third equation in (5.16) we find

$$
\left(\kappa-\mathcal{C}^{T} \mathcal{H}^{-1} \mathcal{C}\right)^{-1}=\left(\kappa+\mathcal{C}^{T} \eta \mathcal{C}\right)^{-1}=\frac{1}{2} \kappa^{-1}\left(1+\widetilde{\mathcal{N}} \kappa^{-1}\right)=\kappa^{-1}+\frac{1}{2} a G^{-1} a^{T} .
$$

This shows that the inverse matrix on the left-hand side always exists for finite $a_{m}{ }^{\alpha}$ and that the bounds on $\mathcal{C}$ do not impose extraneous conditions.

Let us now turn to the potential for the scalar fields, which can be obtained from

$$
V=f^{\alpha \beta \gamma} f^{\alpha^{\prime} \beta^{\prime} \gamma^{\prime}}\left[\frac{1}{12} \widetilde{\mathcal{N}}_{\alpha \alpha^{\prime}} \widetilde{\mathcal{N}}_{\beta \beta^{\prime}} \widetilde{\mathcal{N}}_{\gamma \gamma^{\prime}}-\frac{1}{4} \kappa_{\alpha \alpha^{\prime}} \kappa_{\beta \beta^{\prime}} \widetilde{\mathcal{N}}_{\gamma \gamma^{\prime}}+\frac{1}{6} \kappa_{\alpha \alpha^{\prime}} \kappa_{\beta \beta^{\prime}} \kappa_{\gamma \gamma^{\prime}}\right]
$$

Upon replacing $\widetilde{\mathcal{N}}$ with the second equation in (5.10) one may verify that the potential can be brought into the form

$$
\begin{aligned}
V= & f_{\alpha \beta \gamma} f^{\alpha^{\prime} \beta^{\prime} \gamma^{\prime}}\left[\left(\kappa+\mathcal{C}^{T} \eta \mathcal{C}\right)^{-1}\right]^{\alpha \alpha^{\prime \prime}}\left[\left(\kappa+\mathcal{C}^{T} \eta \mathcal{C}\right)^{-1}\right]^{\beta \beta^{\prime \prime}}\left[\left(\kappa+\mathcal{C}^{T} \eta \mathcal{C}\right)^{-1}\right]^{\gamma \gamma^{\prime \prime}} \\
& {\left[\frac{1}{12}\left(\kappa-\mathcal{C}^{T} \eta \mathcal{C}\right)_{\alpha^{\prime \prime} \alpha^{\prime}}\left(\kappa-\mathcal{C}^{T} \eta \mathcal{C}\right)_{\beta^{\prime \prime} \beta^{\prime}}\left(\kappa-\mathcal{C}^{T} \eta \mathcal{C}\right)_{\gamma^{\prime \prime} \gamma^{\prime}}\right.} \\
& -\frac{1}{4}\left(\kappa+\mathcal{C}^{T} \eta \mathcal{C}\right)_{\alpha^{\prime \prime} \alpha^{\prime}}\left(\kappa+\mathcal{C}^{T} \eta \mathcal{C}\right)_{\beta^{\prime \prime} \beta^{\prime}}\left(\kappa-\mathcal{C}^{T} \eta \mathcal{C}\right)_{\gamma^{\prime \prime} \gamma^{\prime}} \\
& \left.+\frac{1}{6}\left(\kappa+\mathcal{C}^{T} \eta \mathcal{C}\right)_{\alpha^{\prime \prime} \alpha^{\prime}}\left(\kappa+\mathcal{C}^{T} \eta \mathcal{C}\right)_{\beta^{\prime \prime} \beta^{\prime}}\left(\kappa+\mathcal{C}^{T} \eta \mathcal{C}\right)_{\gamma^{\prime \prime} \gamma^{\prime}}\right]
\end{aligned}
$$

Expanding and simplifying the terms inside the last square bracket and relabelling the indices in some terms we get

$$
\begin{aligned}
V= & f_{\alpha \beta \gamma} f^{\alpha^{\prime} \beta^{\prime} \gamma^{\prime}}\left[\left(\kappa+\mathcal{C}^{T} \eta \mathcal{C}\right)^{-1} \mathcal{C}^{T} \eta \mathcal{C}\right]^{\alpha} \alpha^{\prime}\left[\left(\kappa+\mathcal{C}^{T} \eta \mathcal{C}\right)^{-1} \mathcal{C}^{T} \eta \mathcal{C}\right]^{\beta} \beta^{\prime} \\
& \cdot\left[\left(\kappa+\mathcal{C}^{T} \eta \mathcal{C}\right)^{-1}\left(\kappa+\frac{1}{3} \mathcal{C}^{T} \eta \mathcal{C}\right)\right]^{\gamma} \gamma^{\prime} .
\end{aligned}
$$


This form makes it manifest that the potential has no constant terms, i.e., there is no cosmological constant, and no terms quadratic in $\mathcal{C}$, i.e., there are no mass terms for vacua in which the scalars have zero expectation value.

Finally, we rewrite the Yang-Mills term using the $O(d, d)$ covariant field variables. Starting from

$$
L_{\mathrm{YM}}=-\frac{1}{4} \widehat{\mathcal{H}}_{\hat{M} \hat{N}} \widehat{\mathcal{F}}^{\mu \nu \hat{M}} \widehat{\mathcal{F}}_{\mu \nu} \hat{N},
$$

we insert the block components of $\widehat{\mathcal{H}}$ according to (5.15) and the components of the field strengths $\widehat{\mathcal{F}}$ according to (5.22). The resulting Yang-Mills term is simplified by introducing the following combination,

$$
\overline{\mathcal{F}}_{\mu \nu}{ }^{M} \equiv \mathcal{F}_{\mu \nu}{ }^{M}-\eta^{M N} C_{N \alpha} \mathcal{F}_{\mu \nu}{ }^{\alpha} .
$$

A straightforward computation then shows that (5.38) can be written as

$$
\begin{aligned}
L_{\mathrm{YM}}= & -\frac{1}{4}\left(\mathcal{H}+2 \mathcal{C}\left(\kappa+\mathcal{C}^{T} \eta \mathcal{C}\right)^{-1} \mathcal{C}^{T}\right)_{M N} \overline{\mathcal{F}}^{\mu \nu M} \overline{\mathcal{F}}_{\mu \nu}{ }^{N}-\frac{1}{2} \mathcal{C}_{M \alpha} \overline{\mathcal{F}}^{\mu \nu M} \mathcal{F}_{\mu \nu}{ }^{\alpha} \\
& -\frac{1}{4}\left(\kappa+\mathcal{C}^{T} \eta \mathcal{C}\right)_{\alpha \beta} \mathcal{F}^{\mu \nu \alpha} \mathcal{F}_{\mu \nu}{ }^{\beta} .
\end{aligned}
$$

By using $\eta \mathcal{C}=-\mathcal{H}^{-1} \mathcal{C}$ and expanding and resumming the geometric series we can also rewrite this as

$$
\begin{aligned}
L_{\mathrm{YM}}= & -\frac{1}{4}\left(\mathcal{H}\left(\mathcal{H}-\mathcal{C} \kappa^{-1} \mathcal{C}^{T}\right)^{-1}\left(\mathcal{H}+\mathcal{C} \kappa^{-1} \mathcal{C}^{T}\right)\right)_{M N} \overline{\mathcal{F}}^{\mu \nu M} \overline{\mathcal{F}}_{\mu \nu}{ }^{N}-\frac{1}{2} \mathcal{C}_{M \alpha} \overline{\mathcal{F}}^{\mu \nu M} \mathcal{F}_{\mu \nu}{ }^{\alpha} \\
& -\frac{1}{4}\left(\kappa+\mathcal{C}^{T} \eta \mathcal{C}\right)_{\alpha \beta} \mathcal{F}^{\mu \nu \alpha} \mathcal{F}_{\mu \nu}{ }^{\beta}
\end{aligned}
$$

We are now ready to assemble the pieces and give the final form of the dimensionally reduced action, thus summarizing our result. The action is written in terms of the field content

$$
\left\{g_{\mu \nu}, b_{\mu \nu}, \phi, A_{\mu}{ }^{\alpha}, A_{\mu}{ }^{M}, \mathcal{H}_{M N}, \mathcal{C}_{M}{ }^{\alpha}\right\} .
$$

Here the first four fields are $O(d, d)$ singlets, and the final three fields transform in $O(d, d)$ tensor representations. We use matrix notation: $\mathcal{H}$ for $\mathcal{H}_{M N}$ and $\mathcal{C}$ for $\mathcal{C}_{M \alpha}$. The matrix $\mathcal{H}$ satisfies the familiar constraint of the generalized metric $\mathcal{H} \eta \mathcal{H}=\eta$, while $\mathcal{C}$ is constrained by $(1+\mathcal{H} \eta) \mathcal{C}=0$. In terms of these variables and using matrix notation the action reads

$$
\begin{aligned}
S=\int d^{n} x \sqrt{-g} e^{-2 \phi}( & R(g)+4 \partial^{\mu} \phi \partial_{\mu} \phi-\frac{1}{12} H^{\mu \nu \rho} H_{\mu \nu \rho}+\frac{1}{8} \operatorname{Tr}\left(\partial^{\mu} \mathcal{H} \partial_{\mu} \mathcal{H}^{-1}\right) \\
& -\operatorname{Tr}\left[\left(\mathcal{H}-\mathcal{C} \kappa^{-1} \mathcal{C}^{T}\right)^{-1} D^{\mu} \mathcal{C} \mathcal{K}_{\mathcal{C}}^{-1} D_{\mu} \mathcal{C}^{T}\right]-V(\mathcal{C}) \\
& -\frac{1}{4}\left(\mathcal{H}\left(\mathcal{H}-\mathcal{C} \kappa^{-1} \mathcal{C}^{T}\right)^{-1}\left(\mathcal{H}+\mathcal{C} \kappa^{-1} \mathcal{C}^{T}\right)\right)_{M N} \overline{\mathcal{F}}^{\mu \nu M} \overline{\mathcal{F}}_{\mu \nu}{ }^{N} \\
& \left.-\frac{1}{2} \mathcal{C}_{M \alpha} \overline{\mathcal{F}}^{\mu \nu M} \mathcal{F}_{\mu \nu}{ }^{\alpha}-\frac{1}{4}\left(\mathcal{K}_{\mathcal{C}}\right)_{\alpha \beta} \mathcal{F}^{\mu \nu \alpha} \mathcal{F}_{\mu \nu}{ }^{\beta}\right)
\end{aligned}
$$

where we defined the $\mathcal{C}$ dependent extension $\mathcal{K}_{\mathcal{C}}$ of the Cartan-Killing metric:

$$
\mathcal{K}_{\mathcal{C}} \equiv \kappa-\mathcal{C}^{T} \mathcal{H}^{-1} \mathcal{C}=\kappa+\mathcal{C}^{T} \eta \mathcal{C} .
$$


The potential $V(\mathcal{C})$ is given by $(5.37)$,

$$
V=f_{\alpha \beta \gamma} f^{\alpha^{\prime} \beta^{\prime} \gamma^{\prime}}\left[\left(\mathcal{K}_{\mathcal{C}}\right)^{-1} \mathcal{C}^{T} \eta \mathcal{C}\right]^{\alpha}{ }_{\alpha^{\prime}}\left[\left(\mathcal{K}_{\mathcal{C}}\right)^{-1} \mathcal{C}^{T} \eta \mathcal{C}\right]^{\beta}{ }_{\beta^{\prime}}\left[\left(\mathcal{K}_{\mathcal{C}}\right)^{-1}\left(\kappa+\frac{1}{3} \mathcal{C}^{T} \eta \mathcal{C}\right)\right]^{\gamma} \gamma^{\prime}
$$

the 3-form curvature takes the form

$$
H_{\mu \nu \rho}=3\left(\partial_{[\mu} b_{\nu \rho]}-A_{[\mu}{ }^{M} \partial_{\nu} A_{\rho] M}-A_{[\mu}^{\alpha} \partial_{\nu} A_{\rho] \alpha}-\frac{1}{3} f_{\alpha \beta \gamma} A_{\mu}{ }^{\alpha} A_{\nu}{ }^{\beta} A_{\rho}^{\gamma}\right),
$$

and the field strengths and covariant derivatives are

$$
\begin{aligned}
\overline{\mathcal{F}}_{\mu \nu}{ }^{M} & \equiv \mathcal{F}_{\mu \nu}{ }^{M}-\eta^{M N} C_{N \alpha} \mathcal{F}_{\mu \nu}{ }^{\alpha}, \\
\mathcal{F}_{\mu \nu}{ }^{M} & \equiv \partial_{\mu} A_{\nu}{ }^{M}-\partial_{\nu} A_{\mu}{ }^{M} \\
\mathcal{F}_{\mu \nu}{ }^{\alpha} & \equiv 2 \partial_{[\mu} A_{\nu]}{ }^{\alpha}+f^{\alpha}{ }_{\beta \gamma} A_{\mu}{ }^{\beta} A_{\nu}{ }^{\gamma}, \\
D_{\mu} \mathcal{C}_{M \alpha} & \equiv \partial_{\mu} \mathcal{C}_{M \alpha}-A_{\mu}{ }^{\gamma} f_{\gamma \alpha}{ }^{\beta} \mathcal{C}_{M \beta} .
\end{aligned}
$$

This form of the action is manifestly $O(d, d)$ invariant as it is written in terms of $O(d, d)$ covariant objects, with all indices properly contracted. Since both $\mathcal{H}$ and $\mathcal{C}$ are constrained, an unconstrained parameterization of these objects is useful. If we parameterize the matrix $\mathcal{H}$ using a symmetric matrix of scalars $\bar{G}$ and an antisymmetric matrix of scalars $B$ :

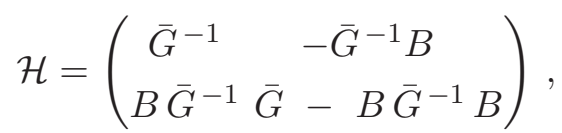

then we can give also an explicit parameterization of $\mathcal{C}$ in terms of a field $A \equiv A_{m \alpha}$ :

$$
\mathcal{C}=\frac{1}{2}\left(\begin{array}{c}
-\bar{G}^{-1} A \\
-B \bar{G}^{-1} A+A
\end{array}\right) .
$$

We can then view $\bar{G}, B$, and $A$ as independent fields. The connection to the original supergravity variables yields a slightly different and more complex parameterization in which $A$ above is set equal to $a^{T} \kappa$ and $\bar{G}$ is set equal to $G+\frac{1}{2} a^{T} \kappa a$, as one can recall from (5.17) and (5.19).

The results of this section apply also if the original higher-dimensional gauge group is of the form $G^{\prime} \times \mathrm{U}(1)^{p}$, with $G^{\prime}$ compact semisimple and of dimension $K^{\prime}$. As we discussed at the end of the previous section, the reduced theory is formally $O\left(d, d+p+K^{\prime}\right)$ invariant but the true symmetry is only $O(d, d+p)$. This time we want to write the theory in terms of $O(d, d+p)$ multiplets. The analysis in this case is a straightfoward generalization of the analysis of this section. Following the discussion of this situation around (4.16), the matrix $\widehat{\mathcal{H}}$ in (5.3) takes now a similar form

$$
\widehat{\mathcal{H}}_{\hat{M} \hat{N}}=\left(\begin{array}{cc}
\widetilde{\mathcal{H}}_{M N} & \widetilde{\mathcal{C}}_{M \beta} \\
\left(\widetilde{\mathcal{C}}^{T}\right)_{\alpha N} & \widetilde{\mathcal{N}}_{\alpha \beta}
\end{array}\right),
$$

but the matrix dimensions are now as follows

$$
\begin{aligned}
\widehat{\eta}, \widehat{\mathcal{H}} & :\left(2 d+p+K^{\prime}\right) \times\left(2 d+p+K^{\prime}\right), \\
\eta, \widetilde{\mathcal{H}} & :(2 d+p) \times(2 d+p), \\
\widetilde{\mathcal{C}} & :(2 d+p) \times K^{\prime}, \\
\kappa^{\prime}, \widetilde{\mathcal{N}} & : K^{\prime} \times K^{\prime} .
\end{aligned}
$$


$\eta$ will now correspond to the matrix

$$
\eta=\left(\begin{array}{ccc}
0 & I_{d} & 0 \\
I_{d} & 0 & 0 \\
0 & 0 & I_{p}
\end{array}\right) .
$$

The analysis proceeds as before. We parameterize $\widetilde{\mathcal{H}}, \widetilde{\mathcal{C}}$, and $\widetilde{\mathcal{N}}$, with matrices $\mathcal{H}$ and $\mathcal{C}$ of sizes

$$
\begin{aligned}
\mathcal{H} & :(2 d+p) \times(2 d+p), \\
\mathcal{C} & :(2 d+p) \times K^{\prime},
\end{aligned}
$$

that satisfy the same constraints as before $(\mathcal{H} \eta \mathcal{H}=\eta,(1+\mathcal{H} \eta) \mathcal{C}=0)$, and thus have the same solutions. As a result, the action takes the form (5.43) with $\mathcal{H} \eta^{-1}$ an $O(d, d+p)$ matrix and $\mathcal{C}$ an $O(d, d+p)$ vector valued in the Lie algebra of $G^{\prime}$. Finally, $\kappa$ is set equal to the Cartan-Killing metric $\kappa^{\prime}$ of the Lie algebra of $G^{\prime}$.

\section{Conclusions}

In this paper we have revisited the effective action of heterotic string theory on a torus and its duality symmetries. The seminal work of Maharana-Schwarz exhibited a global $O(d, d+16 ; \mathbb{R})$ symmetry in the reduction of heterotic supergravity truncated to the Cartan subalgebra on a $d$-torus. This is the proper effective theory for the situation in which the background fields corresponding to the metric $g, b$-field and the gauge fields of the Cartan subalgebra all have non-trivial values. In fact, in this case the non-Cartan parts of the gauge groups $\mathrm{SO}(32)$ or $E_{8} \times E_{8}$ are 'Higgsed' and hence massive. Therefore they have to be ignored in the massless effective action, giving the theory with global $O(d, d+16 ; \mathbb{R})$ symmetry constructed by Maharana-Schwarz. However, we may also consider the situation for which only $g$ and $b$ have non-trivial background values. In this case the full gauge fields corresponding to $\mathrm{SO}(32)$ or $E_{8} \times E_{8}$ remain massless, and so the question arises what is the global duality symmetry upon including all these non-abelian gauge fields. We investigated this question, showed that the duality symmetry is $O(d, d ; \mathbb{R})$ in general and exhibited this symmetry in the novel effective action (5.43) in manifest form. Interestingly, such a formulation requires non-polynomial couplings in the $O(d, d)$ covariant fields as is manifest, for instance, in the form of the potential (5.37).

So far we have displayed the global $O(d, d ; \mathbb{R})$ symmetry of the two-derivative reduced effective theory. The arguments in section 2 show, however, that this continuous symmetry is preserved by arbitrary $\alpha^{\prime}$ corrections. How do we exhibit this symmetry to higher orders in $\alpha^{\prime}$ ? To first order in $\alpha^{\prime}$ a natural possibility is suggested by the results of Bergshoeff and de Roo [36], as recently used in double field theory [24]. They noted that the $\mathcal{O}\left(\alpha^{\prime}\right)$ Riemann-squared corrections can be introduced by treating the torsionful spin connections

$$
\hat{\omega}_{\hat{\mu} \hat{a} \hat{b}}^{(-)}(\hat{e}, \hat{b}) \equiv \hat{\omega}_{\hat{\mu} \hat{a} \hat{b}}(\hat{e})-\frac{1}{2} \hat{H}_{\hat{\mu} \hat{\nu} \hat{\rho}} \hat{e}_{\hat{a}} \hat{\nu}^{\hat{e}_{\hat{b}}} \hat{\rho},
$$

on the same footing as the $\mathrm{SO}(32)$ or $E_{8} \times E_{8}$ gauge fields. These spin connections transform like the gauge fields under supersymmetry and enter the action in the same way: 
with Chern-Simons-type modifications of the three-form field strength and a Yang-Mills term that for the Lorentz connection encodes a Riemann-squared term. The coefficient of the Lorentz Chern-Simons term, however, is opposite to that for the Yang-Mills ChernSimons term. Thus, we may simply include the $\mathcal{O}\left(\alpha^{\prime}\right)$ corrections by formally extending the gauge group to include the Lorentz group, with $\kappa$ chosen to be the negative of the Cartan-Killing metric

$$
\hat{\kappa}_{\hat{a} \hat{b}, \hat{c} \hat{d}}=-\frac{1}{2} \alpha^{\prime} \hat{\eta}_{\hat{a}[\hat{c}} \hat{\eta}_{\hat{d}] \hat{b}} .
$$

Now our formulas of section 5 apply also for this case. There is, however, an important subtlety: the definition (6.1) means we cannot treat $\hat{\omega}^{(-)}$as an independent gauge field. In particular we cannot assign to it independent $O(d, d)$ transformations, as would follow by taking $\hat{\omega}^{(-)}$to be part of an extended generalized metric or constrained $\mathcal{C}$ field. Rather, its $O(d, d)$ transformations to lowest order are fixed by those of $\hat{e}$ and $\hat{b}$, and it needs to be verified that these transformations are compatible. While this is very likely the case, given the checks performed in $[24,32]$, it would be desirable to have a formalism in which this is manifest. This may also shed a new light on the double field theory formulations including higher derivative $\mathcal{O}\left(\alpha^{\prime}\right)$ corrections.

\section{Acknowledgments}

We thank Oscar Bedoya, Eric Bergshoeff, Diego Marques, Henning Samtleben and Misha Vasiliev for useful discussions. The work of O.H. is supported by a DFG Heisenberg fellowship. The work of A.S. was supported in part by the DAE project 12-R\&D-HRI-5.02-0303 and J.C. Bose fellowship of the Department of Science and Technology, India. The work of B.Z. and O.H. is supported by the U.S. Department of Energy (DoE) under the cooperative research agreement DE-FG02-05ER41360.

Open Access. This article is distributed under the terms of the Creative Commons Attribution License (CC-BY 4.0), which permits any use, distribution and reproduction in any medium, provided the original author(s) and source are credited.

\section{References}

[1] K.S. Narain, New Heterotic String Theories in Uncompactified Dimensions $<10$, Phys. Lett. B 169 (1986) 41 [INSPIRE].

[2] K.S. Narain, M.H. Sarmadi and E. Witten, A Note on Toroidal Compactification of Heterotic String Theory, Nucl. Phys. B 279 (1987) 369 [InSPIRE].

[3] G. Veneziano, Scale factor duality for classical and quantum strings, Phys. Lett. B 265 (1991) 287 [INSPIRE].

[4] K.A. Meissner and G. Veneziano, Symmetries of cosmological superstring vacua, Phys. Lett. B 267 (1991) 33 [INSPIRE].

[5] A. Sen, $O(d) \times O(d)$ symmetry of the space of cosmological solutions in string theory, scale factor duality and two-dimensional black holes, Phys. Lett. B 271 (1991) 295 [INSPIRE]. 
[6] S.F. Hassan and A. Sen, Twisting classical solutions in heterotic string theory, Nucl. Phys. B 375 (1992) 103 [hep-th/9109038] [INSPIRE].

[7] J. Maharana and J.H. Schwarz, Noncompact symmetries in string theory, Nucl. Phys. B 390 (1993) 3 [hep-th/9207016] [INSPIRE].

[8] M.J. Duff, B.E.W. Nilsson and C.N. Pope, Kaluza-Klein Approach to the Heterotic String, Phys. Lett. B 163 (1985) 343 [inSPIRE].

[9] M.J. Duff, B.E.W. Nilsson, N.P. Warner and C.N. Pope, Kaluza-Klein Approach to the Heterotic String II, Phys. Lett. B 171 (1986) 170 [INSPIRE].

[10] W. Siegel, Superspace duality in low-energy superstrings, Phys. Rev. D 48 (1993) 2826 [hep-th/9305073] [INSPIRE].

[11] W. Siegel, Manifest duality in low-energy superstrings, hep-th/9308133 [INSPIRE].

[12] C. Hull and B. Zwiebach, Double Field Theory, JHEP 09 (2009) 099 [arXiv:0904.4664] [INSPIRE].

[13] O. Hohm, C. Hull and B. Zwiebach, Background independent action for double field theory, JHEP 07 (2010) 016 [arXiv: 1003.5027] [INSPIRE].

[14] O. Hohm, C. Hull and B. Zwiebach, Generalized metric formulation of double field theory, JHEP 08 (2010) 008 [arXiv: 1006.4823] [INSPIRE].

[15] O. Hohm and S.K. Kwak, Double Field Theory Formulation of Heterotic Strings, JHEP 06 (2011) 096 [arXiv:1103.2136] [INSPIRE].

[16] O. Hohm and S.K. Kwak, $N=1$ Supersymmetric Double Field Theory, JHEP 03 (2012) 080 [arXiv:1111.7293] [INSPIRE].

[17] D. Baraglia and P. Hekmati, Transitive Courant Algebroids, String Structures and T-duality, arXiv:1308.5159 [INSPIRE].

[18] M. Garcia-Fernandez, Torsion-free generalized connections and Heterotic Supergravity, Commun. Math. Phys. 332 (2014) 89 [arXiv:1304.4294] [INSPIRE].

[19] L.B. Anderson, J. Gray and E. Sharpe, Algebroids, Heterotic Moduli Spaces and the Strominger System, JHEP 07 (2014) 037 [arXiv: 1402.1532] [INSPIRE].

[20] X. de la Ossa and E.E. Svanes, Holomorphic Bundles and the Moduli Space of $N=1$ Supersymmetric Heterotic Compactifications, JHEP 10 (2014) 123 [arXiv:1402.1725] [INSPIRE].

[21] O. Hohm, W. Siegel and B. Zwiebach, Doubled $\alpha^{\prime}$-geometry, JHEP 02 (2014) 065 [arXiv:1306.2970] [INSPIRE].

[22] O. Hohm and B. Zwiebach, Green-Schwarz mechanism and $\alpha^{\prime}$-deformed Courant brackets, JHEP 01 (2015) 012 [arXiv:1407.0708] [INSPIRE].

[23] O. Hohm and B. Zwiebach, Double field theory at order $\alpha^{\prime}$, JHEP 11 (2014) 075 [arXiv: 1407.3803] [INSPIRE].

[24] O.A. Bedoya, D. Marques and C. Núñez, Heterotic $\alpha^{\prime}$-corrections in Double Field Theory, JHEP 12 (2014) 074 [arXiv: 1407.0365] [INSPIRE].

[25] A. Coimbra, R. Minasian, H. Triendl and D. Waldram, Generalised geometry for string corrections, JHEP 11 (2014) 160 [arXiv:1407.7542] [INSPIRE]. 
[26] X. de la Ossa and E.E. Svanes, Connections, Field Redefinitions and Heterotic Supergravity, JHEP 12 (2014) 008 [arXiv: 1409.3347] [INSPIRE].

[27] R. Blumenhagen and R. Sun, T-duality, Non-geometry and Lie Algebroids in Heterotic Double Field Theory, arXiv:1411.3167 [INSPIRE].

[28] N. Berkovits, Y. Okawa and B. Zwiebach, WZW-like action for heterotic string field theory, JHEP 11 (2004) 038 [hep-th/0409018] [INSPIRE].

[29] I. Jeon, K. Lee and J.-H. Park, Double field formulation of Yang-Mills theory, Phys. Lett. B 701 (2011) 260 [arXiv:1102.0419] [INSPIRE].

[30] N. Kaloper and R.C. Myers, The O(dd) story of massive supergravity, JHEP 05 (1999) 010 [hep-th/9901045] [INSPIRE].

[31] H. Lü, C.N. Pope and E. Sezgin, Group reduction of heterotic supergravity, Nucl. Phys. B 772 (2007) 205 [hep-th/0612293] [INSPIRE].

[32] E. Bergshoeff, B. Janssen and T. Ortín, Solution generating transformations and the string effective action, Class. Quant. Grav. 13 (1996) 321 [hep-th/9506156] [INSPIRE].

[33] M. Serone and M. Trapletti, A note on T-duality in heterotic string theory, Phys. Lett. B 637 (2006) 331 [hep-th/0512272] [INSPIRE].

[34] C.M. Hull and P.K. Townsend, World Sheet Supersymmetry and Anomaly Cancellation in the Heterotic String, Phys. Lett. B 178 (1986) 187 [INSPIRE].

[35] D. Friedan, E.J. Martinec and S.H. Shenker, Conformal Invariance, Supersymmetry and String Theory, Nucl. Phys. B 271 (1986) 93 [INSPIRE].

[36] E.A. Bergshoeff and M. de Roo, The Quartic Effective Action of the Heterotic String and Supersymmetry, Nucl. Phys. B 328 (1989) 439 [INSPIRE]. 